hep-th/0505192

HUTP-05/A0022

May 2005

\title{
A Vertex Formalism for Local Ruled Surfaces
}

\author{
Duiliu-Emanuel Diaconescu ${ }^{b}$, Bogdan Florea ${ }^{b}$ and Natalia Saulina ${ }^{\sharp}$ \\ b Department of Physics and Astronomy, Rutgers University, \\ Piscataway, NJ 08854-0849, USA \\ \# Jefferson Physical Laboratory, Harvard University, \\ Cambridge, MA 02138, USA
}

\begin{abstract}
We develop a vertex formalism for topological string amplitudes on ruled surfaces with an arbitrary number of reducible fibers embedded in a Calabi-Yau threefold. Our construction is based on large $N$ duality and localization with respect to a degenerate torus action. We also discuss potential generalizations of our formalism to a broader class of Calabi-Yau threefolds using the same underlying principles.
\end{abstract}




\section{Introduction}

Recent developments in Gromov-Witten theory have clearly emphasized the role of localization in understanding the structure of the topological string expansion. For Calabi-Yau threefolds, which are of most physical interest, localization has maximum impact in the local toric case. The topological partition function can be computed exactly to all orders in the genus expansion using the topological vertex formalism [2]. The topological vertex has been given an enumerative interpretation based on localization with respect to a torus action in $[12,18-21]$. The mathematical construction makes it clear that the vertex formalism is well adapted to target spaces which admit a nondegenerate torus action ${ }^{1}$.

However there are many interesting classes of Calabi-Yau threefolds which do not admit such an action. The most prominent examples are compact hypersurfaces in toric varieties, or in the local case, line bundles over del Pezzo surfaces $d P_{k}, k \geq 4$. In such situations, there is no vertex formalism for the topological string partition function.

The purpose of the present paper is to fill out this gap for a certain class of local CalabiYau threefolds which admit a degenerate torus action. More specifically, we will be concerned with torus actions which fix finitely many curves on the threefold. The simplest example in this class is the local theory of a curve constructed in [6].

In [6], the threefold $X$ is the total space of a rank two bundle $N=L_{1} \oplus L_{2}$ over a fixed projective curve $\Sigma$ of genus $g . \quad L_{1}, L_{2}$ are line bundles of degrees $k_{1}, k_{2}$ on $\Sigma$, with $k_{1}+k_{2}=2 g-2$. There is a fiberwise torus action on $X$ with weights $\lambda_{1}, \lambda_{2}$ on $L_{1}, L_{2}$ which fixes the zero section $\Sigma$. The partition function for this class of threefolds has been computed by Bryan and Pandharipande [6] using a TQFT formalism. Their construction has been extended to open string partition functions in $[4,5]$.

In this paper we will consider local Calabi-Yau threefolds isomorphic to the total space of the canonical bundle over a ruled surface $S$ with an arbitrary number of reducible fibers. The surface $S$ is constructed by blowing-up an arbitrary number of points on a projective bundle $\mathbb{P}\left(\mathcal{O}_{\Sigma} \oplus L\right)$ over a curve $\Sigma$ of genus $g$. In order for the resulting threefold to admit a degenerate torus action, we have to blow-up points on the canonical sections of the projective bundle. Our main goal is to find a gluing formalism for topological amplitudes on this class of threefolds. It is worth noting that in special cases, such as ruled surfaces of genus $g \geq 1$ without reducible fibers, the partition function can also be computed using equivariant complex structure deformations. However this is not always the case since such deformations do not exist in general. For example genus zero surfaces with reducible fibers cannot be solved in this manner. Our formalism can be applied uniformly in all cases, and it agrees with the results obtained by complex structure deformations when the later exist.

The paper is structured as follows. The main construction is presented in section two. In section three we outline a string theoretic derivation of our formalism based on large $N$ duality. Section four and the appendix consist of numerical tests of the conjecture.

Acknowledgments. We are very grateful to Robbert Dijkgraaf for sharing his ideas related to gluing negative and positive vertexes with us during the Aspen Workshop on Strings, Branes and Superpotentials, 2004. We also benefited from related discussions with Allan Adams, John McGreevy and David Morrison during the same workshop. We are also grateful

\footnotetext{
${ }^{1}$ For us, a nondegenerate torus action will be a torus action with finitely many isolated fixed points.
} 
to Cumrun Vafa and Mina Aganagic for pointing out an alternative approach to the partition function of $\mathbb{P}^{1} \times \Sigma$ based on ghost branes $[4,5]$ which led to the discussion in section 4.2 . We would also like to thank Ron Donagi, Antonella Grassi and Tony Pantev for collaboration on related projects and mathematical assistance and to Melissa Liu and Greg Moore for reading the manuscript. D.-E.D. would like to acknowledge the support of the Alfred P. Sloan foundation, as well as the hospitality of the Harvard string theory group during the completion of this work. B.F. was supported by DOE grant DE-FG02-96ER40949. N.S. was supported in part by NSF grants PHY-0244821 and DMS-0244464.

\section{The Main Construction}

Let $S=\mathbb{P}\left(\mathcal{O}_{\Sigma} \oplus L\right)$ be a ruled surface over a projective curve $\Sigma$ of genus $g$, where $L$ is a line bundle on $\Sigma$ with $\operatorname{deg}(L) \leq 0$. We will denote by $\Sigma, \Sigma^{\prime}$ the two canonical sections of $S \rightarrow \Sigma$ with intersection numbers

$$
(\Sigma)^{2}=-e, \quad\left(\Sigma^{\prime}\right)^{2}=e, \quad \Sigma \cdot \Sigma^{\prime}=0
$$

where $e=-\operatorname{deg}(L)$. Here we follow the conventions of [14, Chapter V.2. $]^{2}$. Note that the topology of a surface $S$ is completely determined by the integer $e$. From now on we will call $e$ the degree of the surface $S ; g$ is the geometric genus of the surface $S$.

Let $X$ be the total space of the canonical bundle of $S$. We define a torus $T$ action on $X$ with weight $\lambda_{k}$ along the fiber of $K_{S}$ and weight $\lambda_{f}$ along the fibers of $S$. Obviously the fixed loci on $S$ are the two sections $\Sigma, \Sigma^{\prime}$. The topological string partition function can be defined in terms of residual Gromov-Witten theory as in [6]. This means that we sum only over $T$-invariant maps to $X$, which must necessarily factor through the zero section $S$ of $X \rightarrow S$. More precisely, the truncated partition function of the theory is defined by equivariant integration

$$
Z_{\beta}^{\prime}=\sum_{h \in \mathbb{Z}} g_{s}^{2 h-2} \int_{\left[\bar{M}_{h}(S, \beta)\right]_{T}^{v i r}} e_{T}(\mathcal{V})
$$

where $\beta$ is a 2-homology class on $S$ and $\mathcal{V}$ denotes the obstruction complex on the moduli space of genus $h$ stable maps $\bar{M}_{h}(S, \beta)$. This expression clearly depends on the torus weights $\lambda_{k}, \lambda_{f}$. We will be mainly interested in the special case

$$
\lambda_{k}+\lambda_{f}=0
$$

which has no weight dependence. Note that for this special form of the action, the canonical sections $\Sigma, \Sigma^{\prime}$ are both equivariant Calabi-Yau local curves in the language of [6].

We will consider more general examples by introducing reducible fibers in the ruling $S \rightarrow \Sigma$. This can be achieved by blowing-up a certain number of points on the total space of the projective bundle $\mathbb{P}\left(\mathcal{O}_{\Sigma} \oplus L\right)$. In order for the torus action to lift to the blown-up surface, the points should lie on the canonical sections $\Sigma, \Sigma^{\prime}$.

\footnotetext{
${ }^{2}$ We thank S. Katz and B. Szendrői for pointing out an error in the definition of a ruled surface in the first version of the paper.
} 


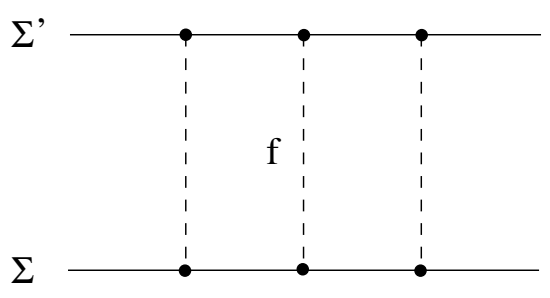

(a)

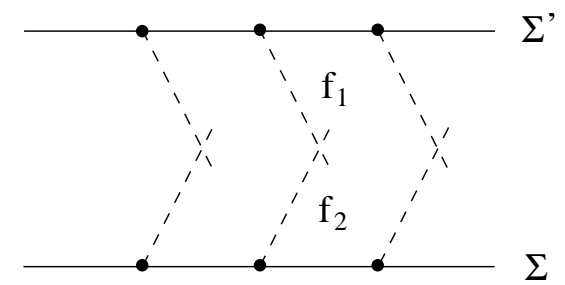

(b)

Figure 1: Ruled surfaces (a) without and (b) with reducible fibers.

The basic building block of our formalism is a trivalent vertex $V$ corresponding to the partition function of the theory for a ruled surface over a genus zero curve with three punctures. This object is indexed by an integer level $p$ which plays a similar role to the level $(a, b)$ in the theory of [6]. We will use the notation ${ }^{p} V$ for the level $p$ vertex. The level zero vertex ${ }^{0} V$ will be often denoted simply by $V$ when the meaning is clear from the context. In principle, such an object should have a rigorous mathematical definition in terms of relative Gromov-Witten invariants, but we will not attempt to make this construction explicit here.

Based on large $N$ duality arguments detailed in the next section, we propose the following construction of the level zero vertex $V$. Each leg of $V$ is labeled by a pair of Young tableaux $\left(R_{i}, R_{i}^{\prime}\right), i=1,2,3$ corresponding to the two canonical sections of $S$ fixed by the torus action. The coefficients $V_{R_{i} R_{i}^{\prime}}$ have a formal expansion of the form

$$
V_{R_{i} R_{i}^{\prime}}=\sum_{d=0}^{\infty} V_{R_{i} R_{i}^{\prime}}^{(d)} q_{f}^{d}
$$

where $q_{f}=e^{-t_{f}}$ is the formal symbol attached to the fiber class. Moreover, $V_{R_{i} R_{i}^{\prime}}^{(d)}$ is zero unless $R_{1}=R_{2}=R_{3}=R, R_{1}^{\prime}=R_{2}^{\prime}=R_{3}^{\prime}=R^{\prime}$. Therefore we can denote the coefficients by $V_{R R^{\prime}}^{(d)}$ keeping in mind that the vertex is trivalent.

Using large $N$ duality, we will show in the next section that $V_{R R^{\prime}}$ is given by the following formula

$$
V_{R R^{\prime}}=\left(\sum_{Q} q_{f}^{l(Q)} W_{R Q} W_{Q R^{\prime}}\right)^{-1}
$$

where $l(Q)$ is the total number of boxes of the Young diagram $Q$ and $W_{R Q}$ are functions of $q=e^{i g_{s}}$ defined in [1] as the large $N$ limit of the S-matrix of Chern-Simons theory

$$
W_{R Q}(q)=\lim _{N \rightarrow \infty} q^{-\frac{N(l(R)+l(Q))}{2}} \frac{S_{R Q}(q, N)}{S_{00}(q, N)} .
$$

Note that $W_{P R}$ is symmetric in $(P, R)$ and can be written in terms of Schur functions $s_{R}$ :

$$
W_{P R}(q)=s_{R}\left(q^{-i+1 / 2}\right) s_{P}\left(q^{R^{i}-i+1 / 2}\right)
$$

where $R^{i}$ is the length of the $\mathrm{i}$-th row of Young diagram $R$ and $i=1, \ldots, \infty$. The important special case of the above expression is

$$
W_{R \bullet}(q)=q^{\kappa(R) / 4} d_{q}(R)
$$


where

$$
d_{q}(R)=\prod_{\square \in R}\left(q^{h(\square) / 2}-q^{-h(\square) / 2}\right)^{-1}
$$

is the quantum dimension of the Young tableau $R . h(\square)$ is the hook length of a given box in the Young tableau $R$ and $\kappa(R)$ is defined by the formula

$$
\kappa(R)=2 \sum_{\square \in R}(i(\square)-j(\square))
$$

where $i(\square), j(\square)$ specify the position of a given box in the Young tableau.

It is curious to note that the right hand side of equation (2.5) is closely related to the quantum dimension of coupled representations of $S U(N)$ introduced in [4, Appendix B]

$$
\operatorname{dim}_{q}\left(R \overline{R^{\prime}}\right)=(-1)^{l(R)+l\left(R^{\prime}\right)} q^{-\frac{\kappa(R)+\kappa\left(R^{\prime}\right)}{2}} q_{f}^{-\frac{l(R)+l\left(R^{\prime}\right)}{2}} \frac{\left(W_{R \bullet} W_{\bullet R^{\prime}}\right)^{2} K_{\bullet \bullet}\left(q_{f}, q\right)}{K_{R R^{\prime}}\left(q_{f}, q\right)}
$$

where $q_{f}$ is related to the rank $N$ of the group $q_{f}=q^{N}$ and [15]

$$
K_{R R^{\prime}}\left(q_{f}, q\right)=\sum_{Q} q_{f}^{l(Q)} W_{R Q} W_{Q R^{\prime}}
$$

By expanding (2.5), we obtain

$$
V_{R R^{\prime}}^{(0)}=\frac{1}{W_{R \bullet} W_{\bullet R^{\prime}}}
$$

which is the square of the level $(0,1)$ pair of pants vertex $P^{(0,1)}$ of [6]. For further reference, note that the level zero vertex can also be written as

$$
V_{R R^{\prime}}=\frac{1}{\left(W_{R \bullet} W_{R^{\prime} \bullet}\right)^{2}} \sum_{Q}(-1)^{l(Q)} W_{R Q} W_{Q^{t} R^{\prime}} q_{f}^{l(Q)}
$$

where $Q^{t}$ denotes the transpose of the Young diagram $Q$. This formula follows from equations (B.8) and (B.9) of [4, Appendix B].

The level $p$ vertex ${ }^{p} V$ is related to ${ }^{0} V$ by the formula

$$
{ }^{p} V_{R R^{\prime}}=\left((-1)^{l\left(R^{\prime}\right)+l(R)} q^{\left(\kappa\left(R^{\prime}\right)-\kappa(R)\right) / 2}\right)^{p}{ }^{0} V_{R R^{\prime}}
$$

For a complete picture we also need to define a cap and a tube. Since a tube can be obtained by a gluing a cap to a pair of pants, it suffices to construct the cap. The cap is the partition function of the theory on a ruled surface over a disk $\Delta$, therefore it is represented by a vertex with one leg labeled by two representations $\left(R, R^{\prime}\right)$. In principle one can have different types of caps depending on the geometry of the central fiber over the disk $\Delta$. To avoid inessential complications, we will restrict ourselves to central fibers with at most two reduced irreducible rational components. Therefore we will have two types of caps $C_{R R^{\prime}}$ and $B_{R R^{\prime}}$ corresponding to a smooth $(0,-2)$ central fiber and to a normal crossing of two $(-1,-1)$ curves respectively. 
Both caps can be determined by localization with respect to a nondegenerate torus action. In addition to the previous action on the fiber, we can let the torus act nontrivially on the base $\Delta$ fixing the origin. Then the cap can be evaluated in the topological vertex formalism of $[2]$. We obtain

$$
\begin{aligned}
C_{R R^{\prime}} & =\sum_{Q} W_{R Q} W_{Q R^{\prime}} q_{f}^{l(Q)} \\
B_{R R^{\prime}} & =\sum_{Q, Q^{\prime}}(-1)^{l(Q)+l\left(Q^{\prime}\right)} q^{-\left(\kappa(Q)+\kappa\left(Q^{\prime}\right)\right) / 2} W_{R Q} W_{Q Q^{\prime}} W_{Q^{\prime} R^{\prime}} q_{f_{1}}^{l(Q)} q_{f_{2}}^{l\left(Q^{\prime}\right)}
\end{aligned}
$$

where $q_{f_{1}}, q_{f_{2}}$ are the formal symbols attached to the irreducible components of the normal crossing in the second case. In the final evaluation of the partition function we will have to impose the relation $q_{f_{1}} q_{f_{2}}=q_{f}$, but in the intermediate steps it is more convenient to think of $q_{f_{1}}, q_{f_{2}}$ as independent variables.

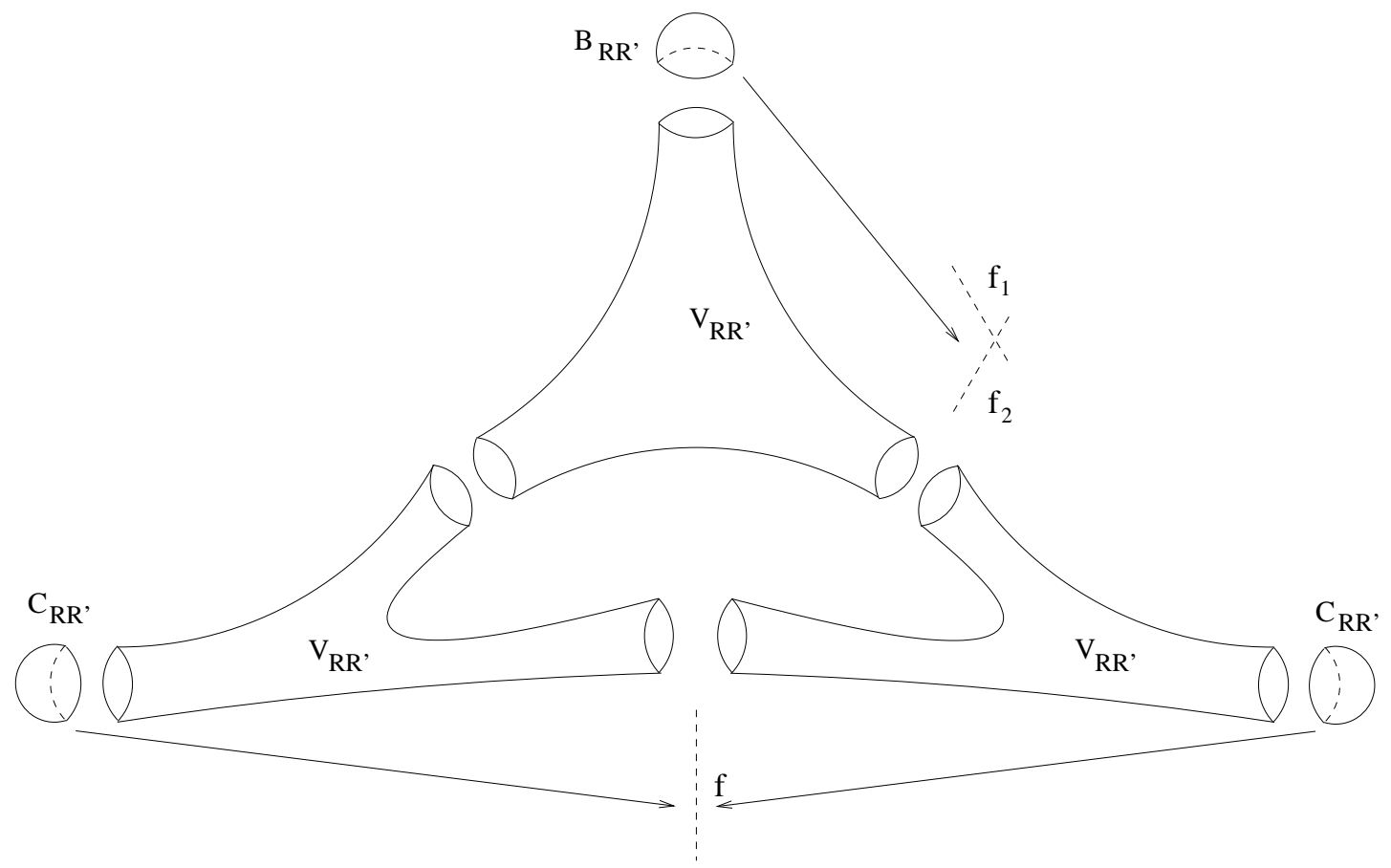

Figure 2: Ruled surface over a genus one curve with one reducible fiber.

Now we can explain how to obtain the partition function of any ruled surface using these building blocks. The simplest case is a surface $S=\Sigma \times \mathbb{P}^{1}$ where $\Sigma$ is a curve of genus $g$. Since $\Sigma$ can be obtained by gluing $2 g-2$ pair of pants, the partition function is simply

$$
Z_{S}=\sum_{R, R^{\prime}}\left(V_{R R^{\prime}}\right)^{2 g-2} q_{b}^{l(R)+l\left(R^{\prime}\right)}
$$

where $q_{b}$ is the formal symbol attached to a section class. Note that the contribution of pure section classes is

$$
\sum_{R, R^{\prime}}\left(V_{R R^{\prime}}^{(0)}\right)^{2 g-2} q_{b}^{l(R)+l\left(R^{\prime}\right)}=\left(\sum_{R}\left(P_{R}^{(0,1)}\right)^{2 g-2} q_{b}^{l(R)}\right)^{2}
$$


where $P_{R}^{(0,1)}$ is the level $(0,1)$ pair of pants of [6]. In the left hand side of equation $(2.16)$ we recognize the square of the partition function of a $(0,2 g-2)$ curve in the equivariant Calabi-Yau case. This represents the contribution of the two canonical sections of $S$ to the partition function. In order to obtain ruled surfaces of degree $e$, we have to glue $2 g-2$ vertexes of the form ${ }^{p} V$ so that the levels add to $e$. This yields the following formula for the degree $e$ partition function

$$
Z_{S}=\sum_{R, R^{\prime}}\left(V_{R R^{\prime}}\right)^{2 g-2} q_{b}^{l(R)} q_{b}^{l\left(R^{\prime}\right)}\left((-1)^{\left(l(R)+l\left(R^{\prime}\right)\right)} q^{\left(\kappa\left(R^{\prime}\right)-\kappa(R)\right) / 2}\right)^{e} .
$$

This algorithm can be generalized to ruled surfaces with reducible fibers by adding caps of the second type. A similar gluing between the local vertex of [6] and the topological vertex was performed in $[4,5]$ for noncompact D-branes in the neighborhood of a local curve. Let $S$ be a ruled surface as above without reducible fibers, $S=\mathbb{P}\left(\mathcal{O}_{\Sigma} \oplus L\right)$. We will denote by $S_{n}$ the blow-up of $S$ at $n$ distinct points $p_{1}, \ldots, p_{n}$ on the canonical section $\Sigma$. Then $S_{n}$ admits a degenerate torus action and we can apply our formalism. In order to obtain the partition function we have to glue together $2 g-2+n$ vertexes and $n$ caps of the second type. We also have to include a correction factor

$$
(-1)^{l(R)} q^{-\kappa(R) / 2}
$$

for each blow-up, taking into account the change in the normal bundle of the canonical section $\Sigma$. The final formula is

$$
Z_{S_{n}}=\sum_{R, R^{\prime}}\left(V_{R R^{\prime}}\right)^{2 g-2+n} q_{b}^{l(R)} q_{b}^{\prime l\left(R^{\prime}\right)}\left((-1)^{\left(l(R)+l\left(R^{\prime}\right)\right)} q^{\left(\kappa\left(R^{\prime}\right)-\kappa(R)\right) / 2}\right)^{e}\left((-1)^{l(R)} q^{-\kappa(R) / 2}\right)^{n}\left(B_{R R^{\prime}}\right)^{n}
$$

We can consider obvious variations of this construction by also blowing-up points on the second canonical section $\Sigma^{\prime}$. The corresponding partition function would be written by analogy with (2.18).

\section{A Large $N$ Duality Derivation}

In this section we will present a string theoretic derivation of the above gluing formalism based on large $N$ duality.

We will start with a specific local Calabi-Yau geometry constructed as follows. Let $S$ denote the Hirzebruch surface $\mathbb{F}_{0}=\mathbb{P}^{1} \times \mathbb{P}^{1}$, and let $\Sigma, \Sigma^{\prime}$ be two fixed $(1,0)$ curves on $S$. For example we can define $\Sigma, \Sigma^{\prime}$ as the fixed loci of a degenerate torus action which acts nontrivially only on one $\mathbb{P}^{1}$ factor. Pick three points $p_{1}, p_{2}, p_{3}$ on $\Sigma$ and respectively $p_{1}^{\prime}, p_{2}^{\prime}, p_{3}^{\prime}$ on $\Sigma^{\prime}$ so that $\left(p_{i}, p_{i}^{\prime}\right)$ belong to the same $(0,1)$ line on $S$ for $i=1,2,3$. Let $\widetilde{S}$ denote the blow-up of $S$ at the points $p_{i}, p_{i}^{\prime}, i=1,2,3 ; \widetilde{S}$ is a nongeneric del Pezzo surface $d P_{7}$ which admits a degenerate torus action. We will denote by $E_{i}, E_{i}^{\prime}, i=1,2,3$ the six exceptional $(-1)$ curves on $\widetilde{S}$. Let $\widetilde{X}$ denote the total space of the canonical bundle over $\widetilde{S}$. Note that $\widetilde{X}$ is a smooth Calabi-Yau threefold and $E_{i}, E_{i}^{\prime}, i=1,2,3$ are $(-1,-1)$ curves on $\widetilde{X}$. 


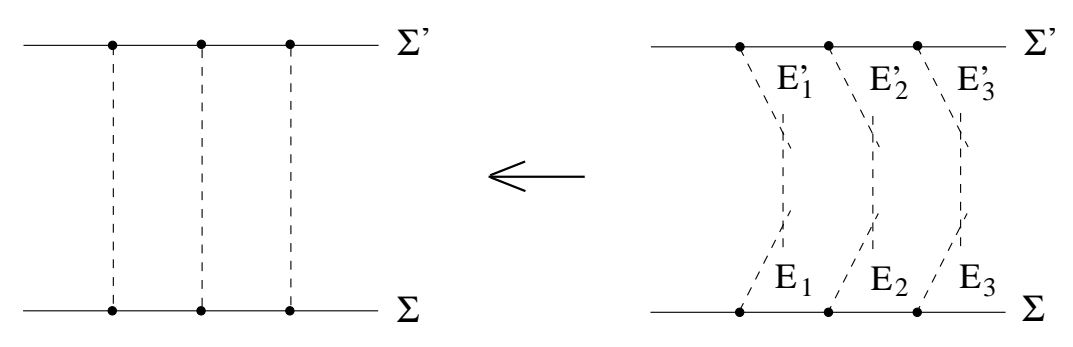

Figure 3: Nongeneric del Pezzo surface $d P_{7}$.

In order to apply large $N$ duality we have to consider an extremal transition of the form

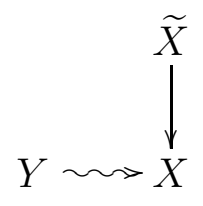

where the vertical arrow is a simultaneous contraction of the six $(-1,-1)$ curves $E_{i}, E_{i}^{\prime}$, $i=1,2,3$ on $\tilde{X}$. The resulting singular threefold $X$ has six isolated ODP's. $Y$ is a generic smoothing of $X$ and the horizontal arrow represents a complex structure degeneration of $Y$ to the singular $X$. In principle there could be global obstructions for such a transition to exist, but in the present case we can explicitly construct a diagram of the form (3.1).

We first describe the singular threefold $X$ as a hypersurface in a rank two bundle over $S=\mathbb{F}_{0}$. Let

$$
\begin{array}{cccc}
Z_{1} & Z_{2} & Z_{3} & Z_{4} \\
1 & 1 & 0 & 0 \\
0 & 0 & 1 & 1
\end{array}
$$

be a toric presentation on $S$. We fix conventions so that the two canonical sections are defined by

$$
\Sigma: Z_{1}=0, \quad \Sigma^{\prime}: Z_{2}=0
$$

Let $Z$ denote the total space of the bundle $\mathcal{O}(-2,1) \oplus \mathcal{O}(0,-2)$ over $S ; Z$ admits the following toric presentation

$$
\begin{array}{cccccc}
Z_{1} & Z_{2} & Z_{3} & Z_{4} & U & V \\
1 & 1 & 0 & 0 & -2 & 0 \\
0 & 0 & 1 & 1 & 1 & -2
\end{array}
$$

where $U, V$ are homogeneous coordinates along the fiber. Consider a hypersurface $X \subset Z$ given by

$$
U Z_{1} Z_{2}+V P_{3}\left(Z_{3}, Z_{4}\right)=0
$$

where $P_{3}\left(Z_{3}, Z_{4}\right)$ is a homogeneous cubic polynomial in $\left(Z_{3}, Z_{4}\right)$. It is easy to check that $X$ is a singular threefold with trivial canonical class. The singularities of $X$ are six isolated ODP's at

$$
\begin{aligned}
& Z_{1}=0, \quad P_{3}\left(Z_{3}, Z_{4}\right)=0 \\
& Z_{2}=0, \quad P_{3}\left(Z_{3}, Z_{4}\right)=0 .
\end{aligned}
$$


The deformation $Y$ is described by adding a linear term in $\left(Z_{3}, Z_{4}\right)$ to the equation (3.4)

$$
U Z_{1} Z_{2}+V P_{3}\left(Z_{3}, Z_{4}\right)=P_{1}\left(Z_{3}, Z_{4}\right)
$$

The coefficients of $P_{1}\left(Z_{3}, Z_{4}\right)$ represent deformation parameters for $Y$. We will assume that $P_{3}\left(Z_{3}, Z_{4}\right)$ and $P_{1}\left(Z_{3}, Z_{4}\right)$ do not have common zeroes so that $Y$ is a generic smooth deformation of the nodal threefold $X$. Using the methods developed in [9-11], one can show that we have six vanishing cycles on $Y$ represented by lagrangian three-spheres $M_{i}, M_{i}^{\prime}$, $i=1,2,3$.

The threefold $\widetilde{X}$ can be obtained performing a simultaneous resolution of the singularities of $X$. In more concrete terms, let $\widetilde{Z}$ be the blow-up of $Z$ along the zero section $U=V=0$. Then $\widetilde{X}$ is the strict transform of $X$ in $\widetilde{Z}$.

Large $N$ duality predicts an equivalence of topological closed string A-model on $\widetilde{X}$ and the topological open-closed A-model on $\left(Y, M_{i}, M_{i}^{\prime}\right)$. The later is constructed by wrapping $N_{i}, N_{i}^{\prime}$ topological A-branes on the spheres $M_{i}, M_{i}^{\prime}, i=1,2,3$. The dynamics of the resulting topological string theory is governed by a cubic string field theory with instanton corrections [27]. The cubic string field theory consists in this case of a Chern-Simons theory with group $U\left(N_{i}\right)$ and respectively $U\left(N_{i}^{\prime}\right)$ localized on the six spheres $M_{i}, M_{i}^{\prime}$. The Chern-Simons theories are coupled by open string instanton effects. These effects are quite hard to sum up explicitly in general situations, but they become more tractable in the presence of a torus action.

The open string instanton corrections were studied in great detail in [9-11], for deformations $Y$ which admit a nondegenerate torus action. Such situations occur for example in the context of geometric transitions for toric Calabi-Yau threefolds. An important lesson we can draw from those computations is that the open string instanton series and the coupling to Chern-Simons theory can be determined by localization with respect to the nondegenerate torus action. In particular all instanton contributions can be obtained by summing over multicovers of T-invariant bordered Riemann surfaces embedded in $Y$ with boundary on the lagrangian spheres. When the T-action is nondegenerate, we have finitely many such objects, and the instanton series can be determined by localization. The coupling with Chern-Simons theory is realized by perturbing the Chern-Simons action by Wilson loop operators attached to the boundaries of T-invariant instantons. If a given sphere $M_{i}$ receives several boundary components $\Gamma_{a}^{(i)}$, forming a knot in $S^{3}$, we have to add a perturbation of the form

$$
\text { (instanton factor) } \times \prod_{a} \operatorname{Tr}\left(U_{a}^{(i)}\right)^{n_{a}^{i}}
$$

where $n_{a}^{i}$ are the winding numbers and $U_{a}^{(i)}$ are the holonomy factors attached to a particular boundary component. This discussion is quite schematic. More details for the nondegenerate case can be found in [9-11].

Let us return to our model. The singular hypersurface $X$ admits a degenerate torus action of the form

$$
Z_{1} \rightarrow t Z_{1}, \quad Z_{2} \rightarrow t Z_{2}, \quad U \rightarrow t^{-2} U
$$

where $t \in \mathbb{C}^{\times}$. The action on the other homogeneous coordinates is trivial. It is easy to check that this action preserves the spheres $M_{i}, M_{i}^{\prime}$, therefore we can try to follow the strategy outlined above in order to determine the instanton corrections. 


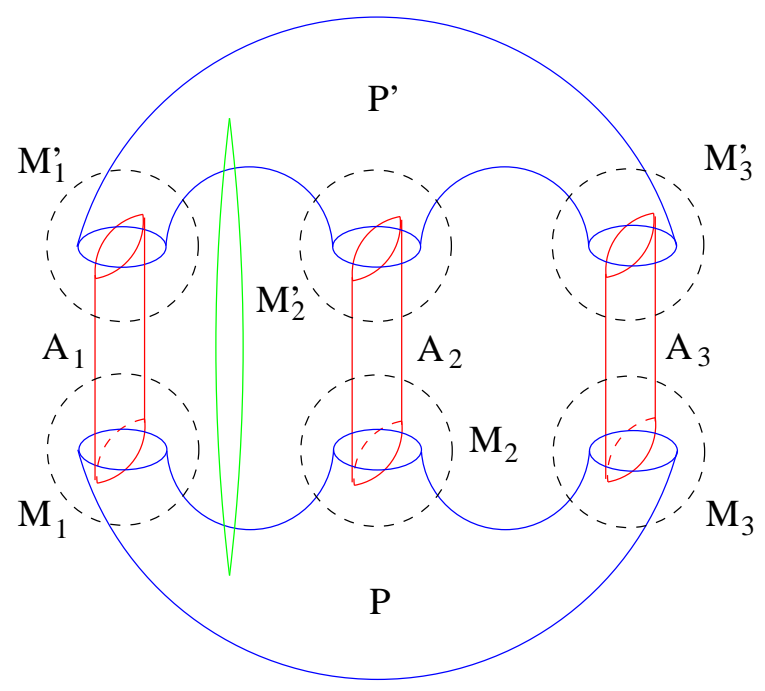

Figure 4: The $d P_{7}$ deformation.

Employing the techniques developed in [9-11], we find the configuration of T-invariant open string surfaces embedded in $Y$ with boundary on $M_{i}, M_{i}^{\prime}, i=1,2,3$. There are two pairs of pants $P, P^{\prime}$ whose boundary components

$$
\partial P=\Gamma_{1}+\Gamma_{2}+\Gamma_{3}, \quad \partial P^{\prime}=\Gamma_{1}^{\prime}+\Gamma_{2}^{\prime}+\Gamma_{3}^{\prime}
$$

are embedded in the six spheres $M_{i}, M_{i}^{\prime}, i=1,2,3$

$$
\Gamma_{i} \subset M_{i}, \quad \Gamma_{i}^{\prime} \subset M_{i}^{\prime} .
$$

$P, P^{\prime}$ are determined by two noncompact rational curves

$$
\begin{aligned}
& P: U=Z_{1}=0, \quad V P_{3}\left(Z_{3}, Z_{4}\right)=P_{1}\left(Z_{3}, Z_{4}\right), \\
& P^{\prime}: U=Z_{2}=0, \quad V P_{3}\left(Z_{3}, Z_{4}\right)=P_{1}\left(Z_{3}, Z_{4}\right) .
\end{aligned}
$$

on $Y$ which intersect the spheres $M_{i}$ and respectively $M_{i}^{\prime}$ along the circles $\Gamma_{i}, \Gamma_{i}^{\prime}$ so that we obtain the configuration in fig. 4. Note that both curves in equation (3.7) are isomorphic to a $\mathbb{P}^{1}$ with three points deleted. The missing points are points at infinity on $Y$ corresponding to the roots of the cubic polynomial $P_{3}\left(Z_{3}, Z_{4}\right)$. We will use the same notation $P, P^{\prime}$ for the punctured $\mathbb{P}^{1}$ 's and the pairs of pants cut by the lagrangian spheres. The distinction should be clear from the context.

We also have three cylinders $A_{i}, i=1,2,3$ connecting the spheres $\left(M_{i}, M_{i}^{\prime}\right), i=1,2,3$ pairwise as in fig. 4. These cylinders are determined by three rational curves on $Y$ with two points deleted given by the equations

$$
V=0, \quad P_{3}\left(Z_{3}, Z_{4}\right)=0, \quad U Z_{1} Z_{2}=P_{1}\left(Z_{3}, Z_{4}\right) .
$$

Note that the above equations describe three curves on $Y$ since $P_{3}\left(Z_{3}, Z_{4}\right)$ has three distinct roots. Each curve intersects a pair of spheres $\left(M_{i}, M_{i}^{\prime}\right)$ along two circles $\Lambda_{i}, \Lambda_{i}^{\prime}, i=1,2,3$ giving rise to the configuration represented in fig. 4 . 
Taking into account all elements found so far, it follows that each sphere $M_{i}$ and each sphere $M_{i}^{\prime}$ receives two boundary components $\Gamma_{i}, \Lambda_{i}$ and respectively $\Gamma_{i}^{\prime}, \Lambda_{i}^{\prime}$ of $T$-invariant open string instantons. $\Gamma_{i}, \Gamma_{i}^{\prime}$ belong to the two pairs of pants $P, P^{\prime}$ and $\Lambda_{i}, \Lambda_{i}^{\prime}$ belong to the three cylinders $A_{i}, i=1,2,3$. Note that in each sphere $\left(\Gamma_{i}, \Lambda_{i}\right)$ and respectively $\left(\Gamma_{i}^{\prime}, \Lambda_{i}^{\prime}\right)$ form a Hopf link with linking number +1 .

The open string instanton corrections associated to this configuration of $T$-invariant surfaces should be determined in principle by summing over all possible multicovers. This is precisely the equivariant enumerative problem solved in [6]. The sum over multicovers of the two pairs of pants represents the pair of pants vertex $P^{(0,1)}$ of [6], while the sum over multicovers of the cylinders corresponds to the tube $A^{(0,0)}$. The novelty here is that these partition functions should be interpreted as corrections to Chern-Simons theory on the six spheres, therefore they have to be coupled to the Chern-Simons action via Wilson loop operators. The net result is that the instanton corrected partition function of the the open-closed topological A-model is of the form

$$
\begin{aligned}
& Z_{Y, M_{i}, M_{i}^{\prime}}^{\text {open }} \\
& =Z_{C S}\left\langle\sum_{R, R^{\prime}} \sum_{Q_{i}} P_{R}^{(0,1)} P_{R^{\prime}}^{(0,1)} q_{b}^{l(R)} q_{b}^{\prime l\left(R^{\prime}\right)} \prod_{i=1}^{3} \operatorname{Tr}_{R}\left(U^{(i)}\right) \operatorname{Tr}_{R^{\prime}}\left(U^{\prime(i)}\right) \operatorname{Tr}_{Q_{i}}\left(V^{(i)}\right) \operatorname{Tr}_{Q_{i}}\left(V^{\prime(i)}\right) q_{i}^{l\left(Q_{i}\right)}\right\rangle
\end{aligned}
$$

Here $R, R^{\prime}$ are Young tableaux attached to the two pairs of pants and $Q_{i}, i=1,2,3$ are Young tableaux attached to the three cylinders. The angular brackets denote the expectation value of Wilson loop operators in Chern-Simons theory. $U^{(i)}$ and respectively $U^{\prime(i)}, i=1,2,3$ are holonomy observables associated to the boundary components of the two pairs of pants. $V^{(i)}$ and $V^{\prime(i)}, i=1,2,3$ are holonomy observables associated to the boundary components of the three cylinders. $q_{b}, q_{b}^{\prime}, q_{i}, i=1,2,3$ are the open string exponentiated Kähler parameter of the pairs of pants $P, P^{\prime}$ and the three cylinders $A_{i}, i=1,2,3$.

Large $N$ duality would predict that the partition function (3.8) should be equal to the closed string partition function $Z_{\widetilde{X}}^{\text {closed }}$ on $\widetilde{X}$ after a suitable change of variables. However it is easy to check that the expression (3.8) is not in agreement to the closed string partition function on the small crepant resolution. In particular it does not yield the correct GromovWitten invariants for fiber classes on $S$. Therefore there is a missing element in the picture developed so far.

In order to understand the missing element, we have to reconsider our computation of the open string instanton corrections. So far we have concentrated on $T$-invariant open string surfaces with boundary on $M_{i}, M_{i}^{\prime}$ trying to mimic the case of nondegenerate torus actions studied in $[9,10]$. However, in the present case, the torus action is degenerate, so we could in principle have nontrivial families of $T$-invariant projective lines on $Y$. In fact since the torus action fixes the punctured curves $P, P^{\prime}$ defined in equation (3.7) pointwise, we expect to find a family of $T$-invariant lines on $Y$ intersecting $P, P^{\prime}$ transversely.

A closer inspection of equations (3.5) shows that $Y$ contains indeed a noncompact ruled surface $F$ determined by the equations

$$
U=0, \quad V P_{3}\left(Z_{3}, Z_{4}\right)=P_{1}\left(Z_{3}, Z_{4}\right) .
$$


From equations (3.9) and (3.7) it follows that the base of the ruling is isomorphic to a rational curve with three points deleted, and $P, P^{\prime}$ are sections of $F$. The fibers of $F$ are smooth compact rational curves on $Y$ with homogeneous coordinates $\left(Z_{1}, Z_{2}\right)$. In fact on can check that $F$ is isomorphic to $\mathbb{F}_{0}$ with three fibers deleted.

In the presence of the ruling $F$ we obtain extra instanton corrections due to open string world-sheets which wrap the fibers of the ruling as well as the pairs of pants $P, P^{\prime}$. From this point of view the present model is similar to the compact transitions studied in [11]. The extra instanton effects will give rise to a series of corrections of the form

$$
V_{R R^{\prime}}=\sum_{d=0}^{\infty} V_{R R^{\prime}}^{(d)} q_{f}^{d}
$$

where $q_{f}=e^{-t_{f}}$ is the exponentiated Kähler area of the fibers of the ruling. The zeroth order term in this expansion is the factor

$$
V_{R R^{\prime}}^{(0)}=P_{R}^{(0,1)} P_{R^{\prime}}^{(0,1)}
$$

already present in (3.8). The higher order terms capture instanton effects with nontrivial degrees along the fibers of the ruling. Note that they have the same coupling to ChernSimons theory as the zeroth order terms since they encode in effect the coupling to the closed string sector.

Then the complete open-closed partition function on $Y$ becomes

$$
\begin{aligned}
& Z_{Y, M_{i}, M_{i}^{\prime}}^{\text {open }} \\
& =Z_{C S}\left\langle\sum_{R, R^{\prime}} \sum_{d=0}^{\infty} \sum_{Q_{i}} V_{R R^{\prime}}^{(d)} q_{f}^{d} q_{b}^{l(R)} q_{b}^{l\left(R^{\prime}\right)} \prod_{i=1}^{3} \operatorname{Tr}_{R}\left(U^{(i)}\right) \operatorname{Tr}_{R^{\prime}}\left(U^{\prime(i)}\right) \operatorname{Tr}_{Q_{i}}\left(V^{(i)}\right) \operatorname{Tr}_{Q_{i}}\left(V^{\prime(i)}\right) q_{i}^{l\left(Q_{i}\right)}\right\rangle
\end{aligned}
$$

Now the main question is how can we determine the higher order coefficients $V_{R R^{\prime}}^{(d)}$. Although this may seem a very hard task at a first look, there is a surprisingly simple solution based on large $N$ duality. The main idea is that there is a refined version of large $N$ duality for noncompact branes which has been extensively used for example in the derivation of the topological vertex [2]. We will employ the same technique in the present case.

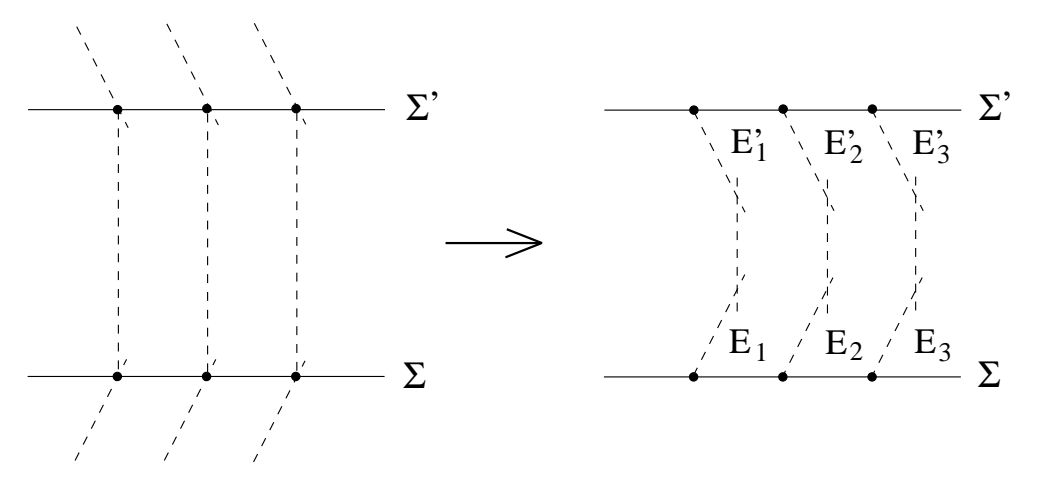

Figure 5: Flopping the exceptional curves. 
Let us first simplify our task by taking a certain limit of the system constructed so far. The limit is easier to describe first on the resolution side of the duality. We will take the area of the exceptional curves $E_{i}, E_{i}^{\prime}, i=1,2,3$ to $-\infty$. This means that we first flop the curves in the threefold $\widetilde{X}$ obtaining a configuration consisting of an $\mathbb{F}_{0}$ surface and six transverse $(-1,-1)$ curves as in fig. 5 . Then we send the volume of the flopped curves to $\infty$, obtaining a local $\mathbb{F}_{0}$ geometry which is toric. A similar limit has been used in the context of large $N$ duality in [1].

On the deformation side, the effect of this limit is to truncate all Chern-Simons expectation values of Hopf links to their leading terms in the power of the 't Hooft coupling. Then the expression (3.12) becomes

$$
Z^{\text {open }}=\sum_{R, R^{\prime}} \sum_{d=0}^{\infty} \sum_{Q_{i}} V_{R R^{\prime}}^{(d)} q_{f}^{d} q_{b}^{l(R)+l\left(R^{\prime}\right)} \prod_{i=1}^{3} W_{R Q_{i}} W_{Q_{i} R^{\prime}} q_{f}^{l\left(Q_{i}\right)} .
$$

In writing (3.13) we have also taken into account the shift in Kähler parameters $q_{i} \rightarrow q_{f}$ found in $[9]$.

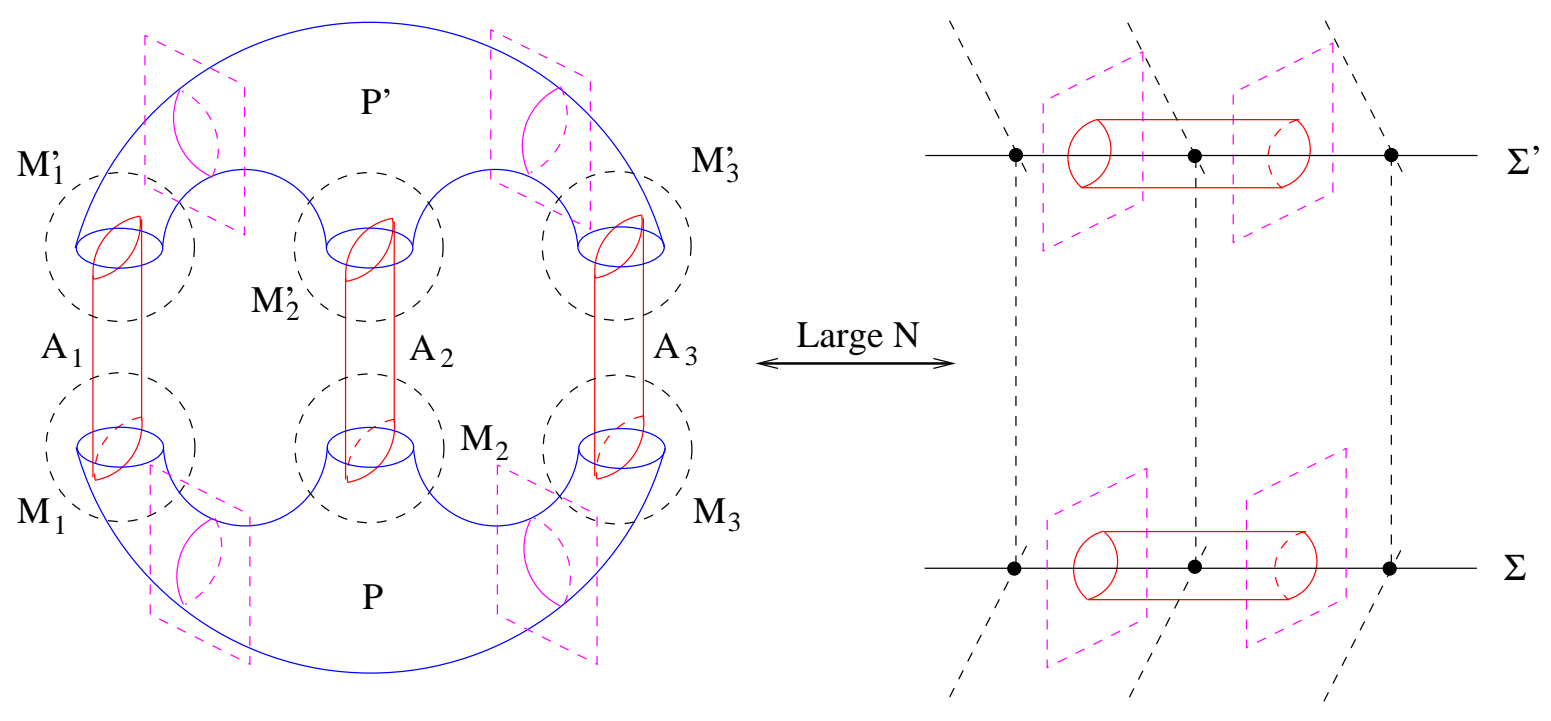

Figure 6: Introducing noncompact branes.

Now we add noncompact branes with topology $S^{1} \times \mathbb{R}^{2}$ to the system which intersect the two pairs of pants as shown in fig. 6. These branes will correspond to similar noncompact branes in the threefold $\widetilde{X}$ intersecting the canonical sections $\Sigma, \Sigma^{\prime}$ as in fig. 6. Large $N$ duality predicts an agreement of topological amplitudes even in the presence noncompact branes. In the limit described above, $\widetilde{X}$ becomes isomorphic to the total space of the canonical bundle over a toric surface $\mathbb{F}_{0}$. Therefore the topological amplitudes for the noncompact branes on the resolution side can be easily computed using the topological vertex.

Let us denote by $T_{1}, T_{2}, T_{1}^{\prime}, T_{2}^{\prime}$ the holonomy observables associated to the noncompact branes. The topological free energy will be a formal series in traces of the form

$$
\operatorname{Tr}_{S_{i}}\left(T_{i}^{d_{i}}\right), \quad \operatorname{Tr}_{S_{i}^{\prime}}\left(\left(T_{i}^{\prime}\right)^{d_{i}^{\prime}}\right)
$$


where $S_{i}, S_{i}^{\prime} i=1,2$ are representations labeled by Young tableaux and $d_{i}, d_{i}^{\prime}, i=1,2$ are the winding numbers associated to the nontrivial 1-cycles on the branes. Here we fix orientations so that open string instantons ending on a noncompact brane from the left have negative winding and open string instantons ending on a noncompact brane from the right have positive winding number.

Before we compare the partition functions with noncompact branes on the two sides of the duality we will take a second limit sending the volume of the sections $\Sigma, \Sigma^{\prime}$ of $\mathbb{F}_{0}$ at infinity. On the resolution side, this leaves us with a very simple system of four noncompact branes joined by two cylinders as in fig. 6. The partition function of this system is simply

$$
\sum_{R, R^{\prime}} q_{o p}^{l(R)+l\left(R^{\prime}\right)} \operatorname{Tr}_{R} T_{1} \operatorname{Tr}_{R} T_{2}^{-1} \operatorname{Tr}_{R^{\prime}} T_{1}^{\prime} \operatorname{Tr}_{R^{\prime}} T_{2}^{\prime-1}
$$

and it represents the sum over multicovers of the two cylinders. Here $q_{o p}$ is the common open string Kähler parameter of the cylinders.

The partition function of the local configuration of branes on the deformation side is given in this limit by

$$
\sum_{R, R^{\prime}} \sum_{d=0}^{\infty} \sum_{Q} V_{R R^{\prime}}^{(d)} q_{f}^{d+l(Q)} W_{R Q} W_{Q R^{\prime}} q_{o p}^{l(R)+l\left(R^{\prime}\right)} \operatorname{Tr}_{R} T_{1} \operatorname{Tr}_{R} T_{2}^{-1} \operatorname{Tr}_{R^{\prime}} T_{1}^{\prime} \operatorname{Tr}_{R^{\prime}} T_{2}^{\prime-1}
$$

Note that $q_{o p}$ is now the common open string Kähler parameter of the two pairs of pants, which is taken equal to the Kähler parameter of the cylinders in (3.14). Note also that the size of the fibers of the ruling $F$ defined in (3.9) is kept finite, hence all the higher degree corrections $V_{R R^{\prime}}^{(d)}$ are present in (3.15).

Large $N$ duality predicts that the two expressions should agree as formal power series in $q_{\text {op }}$ and the holonomy variables $\operatorname{Tr}_{R} T_{1}, \operatorname{Tr}_{R} T_{2}^{-1}, \operatorname{Tr}_{R^{\prime}} T_{1}^{\prime}, \operatorname{Tr}_{R^{\prime}} T_{2}^{\prime-1}$. This implies the following identity of formal power series in $q_{f}$

$$
\sum_{d=0}^{\infty} \sum_{Q} V_{R R^{\prime}}^{(d)} q_{f}^{d+l(Q)} W_{R Q} W_{Q R^{\prime}}=1
$$

for any pair of representations $\left(R, R^{\prime}\right)$. This yields formula $(2.5)$.

One can also derive the normal bundle corrections factors in (2.17) from large $N$ duality. Since level $p$ vertexes can be obtained by gluing together level one vertexes and caps of first type, it suffices to derive the level one vertex.

This can be done using an extremal transition as above in which $\mathbb{F}_{0}$ is replaced by $\mathbb{F}_{1}$. The surface $S$ is now a blow-up of $\mathbb{F}_{1}$ at six points on the canonical fibers and all arguments go through essentially unchanged. In this case we will obtain an identity of the form

$$
\sum_{d=0}^{\infty} \sum_{Q} V_{R R^{\prime}}^{(d)} q_{f}^{d+l(Q)} W_{R Q} W_{Q R^{\prime}}=(-1)^{l(R)+l\left(R^{\prime}\right)} q^{\left(\kappa\left(R^{\prime}\right)-\kappa(R)\right) / 2} .
$$

for the higher degree corrections. This yields again formula (2.13) with $p=1$. 


\section{Experimental Evidence}

Since our construction is not mathematically rigorous, in this section we will perform several numerical tests of the formalism, obtaining exact agreement with enumerative calculations. This is positive evidence for our conjecture, but not a rigorous proof.

\subsection{Genus Zero Surfaces}

The first consistency check of our formalism is agreement with known results for toric ruled surfaces $S$. In particular we should be able to recover the known expressions for the partition function for $S=\mathbb{F}_{0}, \mathbb{F}_{1}$ and their toric blow-ups.

Applying the rules given in section two, the partition function for $S=\mathbb{F}_{e}, e=0,1$ is obtained by gluing a level $e$ pair of pants ${ }^{e} V_{R R^{\prime}}$ and three caps of the first type $C_{R R^{\prime}}$. Then we obtain the following expression

$$
\sum_{R, R^{\prime}} \sum_{Q_{i}} \sum_{d=0}^{\infty}{ }^{e} V_{R R^{\prime}}^{(d)} q_{f}^{d} \prod_{i=1}^{3}\left(W_{R Q_{i}} W_{Q_{i} R^{\prime}} q_{f}^{l\left(Q_{i}\right)}\right) q_{b}^{l(R)} q_{b}^{\prime l\left(R^{\prime}\right)}
$$

This formula can be easily simplified by performing the sum over the fiber degree $d$ and $Q_{3}$ keeping all other Young tableau in the sum fixed. $q_{b}, q_{b}^{\prime}$ denote the exponentiated Kähler parameters of the canonical sections and $q_{f}$ is the exponentiated Kähler parameter of the fiber. Note that $q_{b}^{\prime}=q_{b} q_{f}^{e}, e=0,1$.

Using the formula (2.5), and taking into account the symmetry property $W_{P P^{\prime}}=W_{P^{\prime} P}$ for any $\left(P, P^{\prime}\right)$, this formula becomes

$$
\sum_{R, R^{\prime}} \sum_{Q_{1}, Q_{2}} W_{R Q_{1}} W_{Q_{1} R^{\prime}} W_{R^{\prime} Q_{2}} W_{Q_{2} R} q^{e\left(\kappa\left(R^{\prime}\right)-\kappa(R)\right) / 2}(-1)^{e\left(l(R)+l\left(R^{\prime}\right)\right)} q_{b}^{l(R)} q_{b}^{l\left(R^{\prime}\right)} q_{f}^{l\left(Q_{1}\right)+l\left(Q_{2}\right)} .
$$

This is the partition function of the local $\mathbb{F}_{e}$ model, $e=0,1[1,2,16]$ in the topological vertex formulation. The same computation goes through essentially unchanged for toric blow-ups of $\mathbb{F}_{e}, e=0,1$. The only difference is that one or two caps of the first type are replaced by caps of the second type in equation (4.1), leaving the third cap unchanged. Then we can still sum over $d$ and $Q_{3}$ first and proceed as above, obtaining again the expected result.

A more interesting test can be performed for a blow-up of $\mathbb{F}_{e}, e=0,1$ at three distinct points on the canonical section $\Sigma$. Then we obtain a nontoric surface with partition function

$$
\begin{aligned}
Z=\sum_{R, R^{\prime}} \sum_{Q_{i}, Q_{i}^{\prime}} \sum_{d=0}^{\infty} & { }^{e} V_{R R^{\prime}}^{(d)} q_{f}^{d}(-1)^{l(R)} q^{-3 \kappa(R) / 2} q_{b}^{l(R)} q_{b}^{\prime l\left(R^{\prime}\right)} \\
& \times \prod_{i=1}^{3}\left(W_{R Q_{i}} W_{Q_{i} Q_{i}^{\prime}} W_{Q_{i}^{\prime} R^{\prime}}(-1)^{l\left(Q_{i}\right)+l\left(Q_{i}^{\prime}\right)} q^{-\left(\kappa\left(Q_{i}\right)+\kappa\left(Q_{i}^{\prime}\right)\right) / 2} q_{i}^{l\left(Q_{i}\right)} q_{i}^{l\left(Q_{i}^{\prime}\right)}\right),
\end{aligned}
$$

where $q_{b}, q_{b}^{\prime}, q_{f}$ are Kähler parameters of the two canonical sections and respectively the fiber class and $q_{i}, q_{i}^{\prime}, i=1,2,3$ are Kähler parameters of the irreducible components of the three singular fibers. Note that we have the relations $q_{b}^{\prime}=q_{b} q_{f}^{e}, q_{i} q_{i}^{\prime}=q_{f}$ for $i=1,2,3$. 


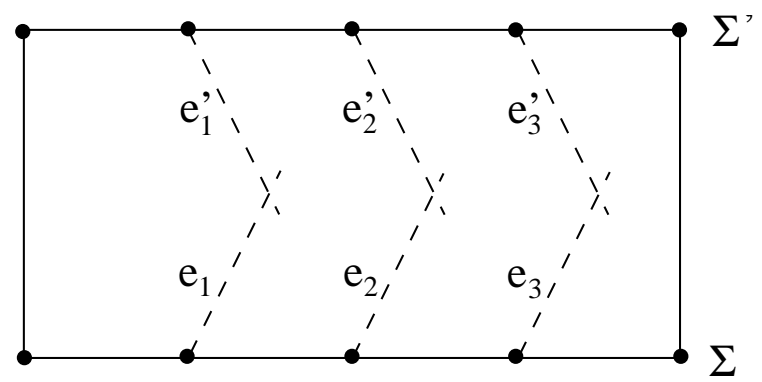

Figure 7: Three point blow-up of $\mathbb{F}_{0}$.

Let us compute the expansion of the free energy $F=\log (Z)$ in powers of the exponentiated Kähler parameters $q_{b}, q_{f}, q_{i}, i=1,2,3$ for $e=0$. This is a very tedious, although fairly straightforward computation, so we will skip the details. The final answer is an expansion of the form

$$
\begin{aligned}
& \frac{1}{\left(2 \sin \frac{g_{s}}{2}\right)^{2}}[-2 q_{b}-2 q_{f}+q_{1}+q_{2}+q_{3}+q_{f}\left(q_{1}^{-1}+q_{2}^{-1}+q_{3}^{-1}\right)+q_{b}\left(q_{1}^{-1}+q_{2}^{-1}+q_{3}^{-1}\right) \\
&\left.-q_{b}\left(q_{1}^{-1} q_{2}^{-1}+q_{2}^{-1} q_{3}^{-1}+q_{3}^{-1} q_{1}^{-1}\right)+q_{b} q_{1}^{-1} q_{2}^{-1} q_{3}^{-1}\right] \\
&+\frac{1}{\left(2 \sin \frac{g_{s}}{2}\right)^{2}}[-4 q_{b} q_{f}+3 q_{b} q_{f}\left(q_{1}^{-1}+q_{2}^{-1}+q_{3}^{-1}\right)-2 q_{b} q_{f}\left(q_{1}^{-1} q_{2}^{-1}+q_{2}^{-1} q_{3}^{-1}+q_{3}^{-1} q_{1}^{-1}\right) \\
&\left.+2 q_{b} q_{f} q_{1}^{-1} q_{2}^{-1} q_{3}^{-1}\right] \\
&+\frac{1}{\left(2 \sin \frac{g_{s}}{2}\right)^{2}}\left[-q_{b}^{2} q_{1}^{-2} q_{2}^{-2} q_{3}^{-2}+q_{b}^{2}\left(q_{1}^{-1} q_{2}^{-2} q_{3}^{-2}+q_{1}^{-2} q_{2}^{-1} q_{3}^{-2}+q_{1}^{-2} q_{2}^{-2} q_{3}^{-1}\right)\right.\left.-q_{b}^{2}\left(q_{1}^{-2} q_{2}^{-1} q_{3}^{-1}+q_{1}^{-1} q_{2}^{-2} q_{3}^{-1}+q_{1}^{-1} q_{2}^{-1} q_{3}^{-2}\right)\right] \\
&+\frac{1}{2\left(2 \sin g_{s}\right)^{2}}\left[-2 q_{b}^{2}-2 q_{f}^{2}+q_{1}^{2}+q_{2}^{2}+q_{3}^{2}+q_{f}^{2}\left(q_{1}^{-2}+q_{2}^{-2}+q_{3}^{-2}\right)+q_{b}^{2}\left(q_{1}^{-2}+q_{2}^{-2}+q_{3}^{-2}\right)\right. \\
&\left.\quad-q_{b}^{2}\left(q_{1}^{-2} q_{2}^{-2}+q_{2}^{-2} q_{3}^{-2}+q_{3}^{-2} q_{1}^{-2}\right)+q_{b}^{2} q_{1}^{-2} q_{2}^{-2} q_{3}^{-2}\right] \\
& \frac{1}{\left(2 \sin \frac{g_{s}}{2}\right)^{2}}\left[2 q_{b}^{2} q_{f}\left(q_{1}^{-2} q_{2}^{-2} q_{3}^{-1}+q_{1}^{-2} q_{2}^{-1} q_{3}^{-2}+q_{1}^{-1} q_{2}^{-2} q_{3}^{-2}\right)+0 q_{b}^{2} q_{f}\left(q_{1}^{-2} q_{2}^{-2}+q_{2}^{-2} q_{3}^{-2}+q_{3}^{-2} q_{1}^{-2}\right)\right. \\
&\left.\quad-2 q_{b}^{2} q_{f} q_{1}^{-2} q_{2}^{-2} q_{3}^{-2}+\cdots\right] \\
&+\cdots .
\end{aligned}
$$

We will show below that the terms computed above provide strong positive evidence for our construction.

First note that the expression (4.4) has the correct BPS expansion of a genus zero topological partition function. In particular the second degree contributions in the third term exhibit the expected multicover behavior. For a quantitative test, note that the coefficient of a monomial of the form $q_{b}^{n_{b}} q_{f}^{n_{f}} q_{1}^{-n_{1}} q_{2}^{-n_{2}} q_{3}^{-n_{3}}$ represents the genus zero Gromov-Witten invariant of a curve class of the form

$$
n_{b} s+n_{f} f-\sum_{i=1}^{3} n_{i} e_{i}
$$


where $(s, f)$ are the section and respectively the fiber class of $\mathbb{F}_{0}$ and $e_{i}, i=1,2,3$ are the exceptional curve classes. If one of the $n_{i}$ is zero, this curve class is pulled back from a toric blow-up of $\mathbb{F}_{0}$ at one or two points. Therefore the Gromov-Witten invariants for such classes can be computed using localization or the topological vertex on surfaces with one less blow-up. Then it is not hard to check that all coefficients of monomials with one $n_{i}=0$ are correct. For example the curve class $a+b-e_{1}$ is a pull-back of the hyperplane class on $\mathbb{P}^{2}$. The corresponding invariant +3 predicted by formula (4.4) is indeed the correct Gromov-Witten invariant for the hyperplane class of $\mathbb{P}^{2}$. The same is true for all classes of this form. This is a nontrivial test of our formalism since the starting point of the expansion - namely equation (4.3) - is qualitatively different from the topological vertex formulas for toric cases. In particular, since all inserted caps are of second type we cannot trivially simplify the expression using the formula (2.5).

The expression (4.4) also yields several predictions for invariants of nontoric curve classes encoded in the terms

$$
\begin{aligned}
& q_{b} q_{1}^{-1} q_{2}^{-1} q_{3}^{-1}-q_{b}^{2} q_{1}^{-2} q_{2}^{-2} q_{3}^{-2}+q_{b}^{2}\left(q_{1}^{-1} q_{2}^{-2} q_{3}^{-2}+q_{1}^{-2} q_{2}^{-1} q_{3}^{-2}+q_{1}^{-2} q_{2}^{-2} q_{3}^{-1}\right)-q_{b}^{2}\left(q_{1}^{-2} q_{2}^{-1} q_{3}^{-1}\right. \\
& \left.+q_{1}^{-1} q_{2}^{-2} q_{3}^{-1}+q_{1}^{-1} q_{2}^{-1} q_{3}^{-2}\right)+2 q_{b} q_{f} q_{1}^{-1} q_{2}^{-1} q_{3}^{-1}+2 q_{b}^{2} q_{f}\left(q_{1}^{-2} q_{2}^{-2} q_{3}^{-1}+q_{1}^{-2} q_{2}^{-1} q_{3}^{-2}+q_{1}^{-1} q_{2}^{-2} q_{3}^{-2}\right) \\
& -2 q_{b}^{2} q_{f} q_{1}^{-2} q_{2}^{-2} q_{3}^{-2}
\end{aligned}
$$

where we have omitted the BPS multicover factors. In appendix A we will check some of these predictions by enumerative computations.

\subsection{Higher Genus Surfaces}

In this subsection, we will test the formalism for higher genus $g \geq 1$ surfaces of the form $S=\Sigma \times \mathbb{P}^{1}$. In this case the partition function is

$$
Z=\sum_{R, R^{\prime}}\left(V_{R R^{\prime}}\right)^{2 g-2} q_{b}^{l(R)+l\left(R^{\prime}\right)} .
$$

In principle, one can test this formula by comparing the free energy term by term with direct enumerative computations. This can be done in practice for low degree terms in the expansion, but we can perform a more convincing test following a different route.

The key observation $[13,17,23-25]$ is that the total space of the canonical bundle of $S$ admits a family of complex structure deformations $X_{\alpha}$ classified by abelian differentials $\alpha \in H^{0}\left(\Sigma, K_{\Sigma}\right)$. Note that these deformations are absent for genus zero surfaces with any number of reducible fibers, so this approach does not apply to that case.

These deformations can be constructed explicitly as follows [13]. First note that a direct product surface $S=\Sigma \times \mathbb{P}^{1}$ can be represented as the projectivization of a rank two bundle of the form $L \oplus L$ on $\Sigma$, where $L$ is a square root of $K_{\Sigma}$. Let $\widehat{X}$ be the singular threefold obtained by contracting the fibers of the ruling on $X ; \widehat{X}$ is isomorphic to the total space of the quotient $L \oplus L /( \pm 1)$, therefore it has $A_{1}$ singularities along the zero section of $L \oplus L \rightarrow \Sigma$. Note that $\widehat{X}$ is isomorphic to a hypersurface in the total space of the bundle $L^{\otimes 2} \oplus L^{\otimes 2} \oplus L^{\otimes 2} \simeq K_{\Sigma}^{\oplus 3}$ on $\Sigma$ with defining equation

$$
U V=W^{2}
$$


One can generate a family of deformations $\widehat{X}_{\alpha}$ of $\widehat{X}$ by perturbing the equation (4.7)

$$
U V=W^{2}-\alpha^{2}
$$

For generic $\alpha \in H^{0}\left(\Sigma, K_{\Sigma}\right), \widehat{X}_{\alpha}$ has $2 g-2$ ordinary double points at

$$
U=V=W=0, \quad \alpha=0 .
$$

These conifold points can be resolved by blowing-up the ambient space along the section $U=W=0$. The strict transform $X_{\alpha}$ of $\widehat{X}_{\alpha}$ is a smooth Calabi-Yau threefold. Moreover, one can show that $X_{\alpha}$ is a family of deformations of $X[13]$.

For us, it is important to note that $X_{\alpha}$ admits a degenerate torus action which fixes finitely many curves. This action is induced by the following action on $\widehat{X}_{\alpha}$

$$
U \rightarrow \lambda U, \quad V \rightarrow \lambda^{-1} V, \quad W \rightarrow W
$$

The fixed locus of this action on $\widehat{X}_{\alpha}$ consists of two genus $g$ curves $\Sigma, \Sigma^{\prime}$ given by equations

$$
U=V=0, \quad W= \pm \alpha
$$

Note that both $\Sigma, \Sigma^{\prime}$ pass through the $2 g-2$ singular points, and have no other common points. The fixed locus of the induced torus action on the resolution is a configuration of curves $\Sigma \cup \Sigma^{\prime} \cup f_{1} \cup \ldots \cup f_{2 g-2}$ where $\Sigma, \Sigma^{\prime}$ are the strict transforms of the curves (4.10) under blow-up and $f_{1}, \ldots, f_{2 g-2}$ are exceptional $(-1,-1)$ curves. Each $f_{m}, m=1, \ldots, 2 g-2$ intersects $\Sigma, \Sigma^{\prime}$ transversely at one point as shown below.

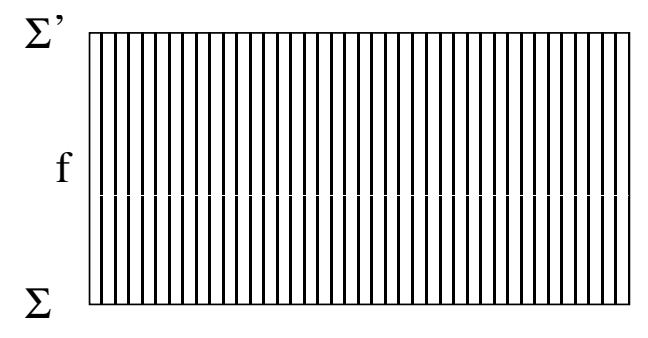

(a)

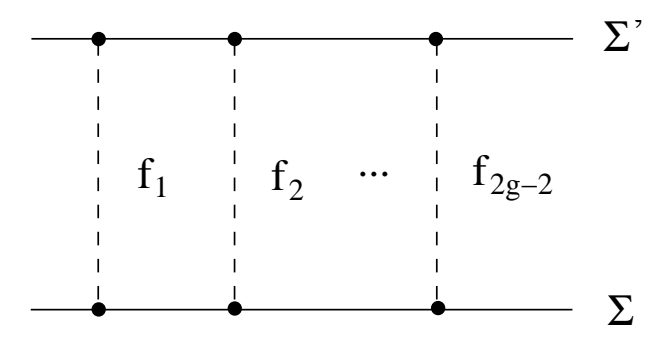

(b)

Figure 8: (a) Ruled surface over a genus $g$ curve $\Sigma$; (b) The deformation.

The partition function for the deformation can then be easily computed by decomposing the curve configuration $\Sigma \cup \Sigma^{\prime} \cup f_{1} \cup \ldots \cup f_{2 g-2}$ into pairs of pants and topological vertexes as shown in fig. 9 for $g=2$. A similar computation has been performed for noncompact D-branes in the neighborhood of a local curve in $[4,5]$.

We have to decompose each of the curves $\Sigma, \Sigma^{\prime}$ into $4 g-4$ level $(0,1)$ pair of pants $P_{R}^{(0,1)}$ and respectively $P_{R^{\prime}}^{(0,1)}$ given by [6]

$$
P_{S}^{(0,1)}=\sum_{S} \frac{1}{W_{S}} .
$$




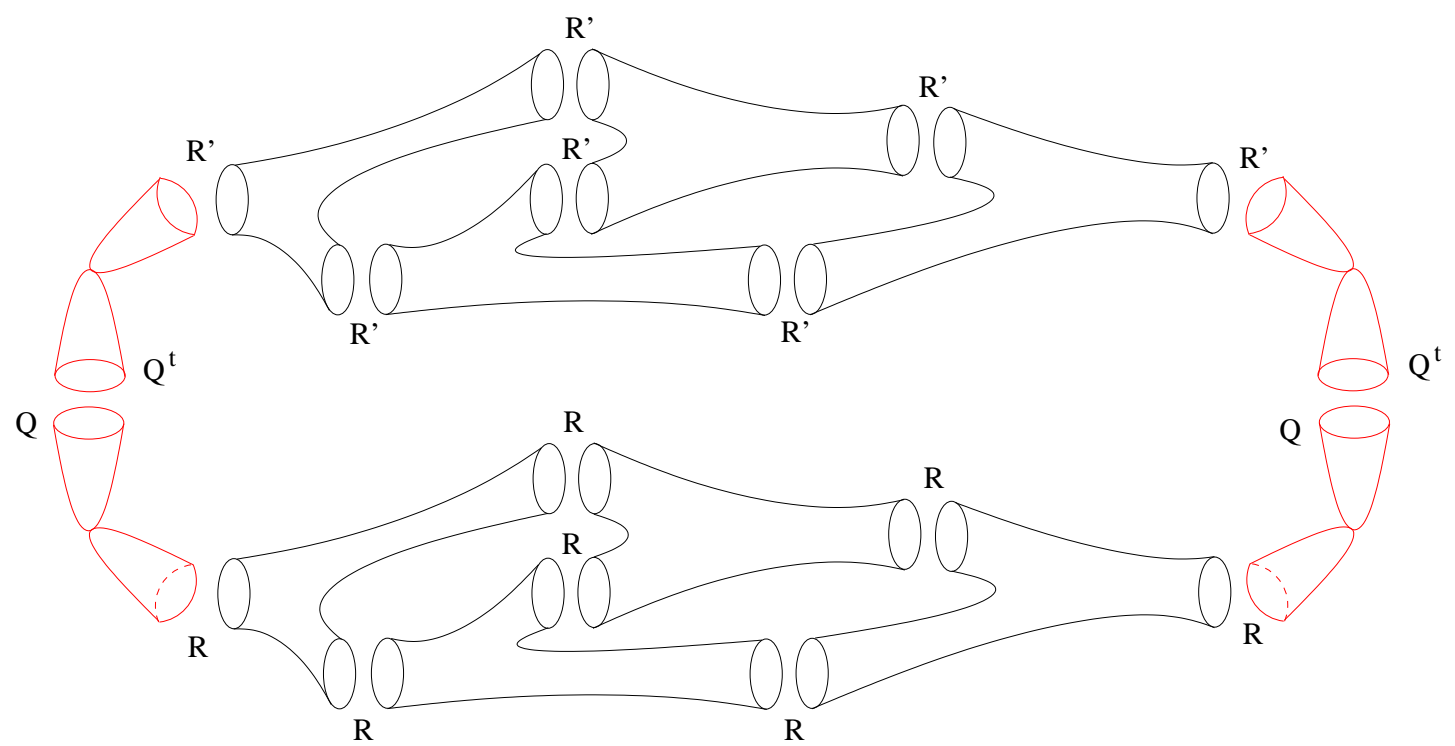

Figure 9: Decomposition of the genus 2 deformation.

We also obtain $(2 g-2)$ pairs of topological vertexes which are glued to the pairs of pants as in fig. 9. Note that the contribution of each such pair equals the topological partition function of a local conifold geometry with noncompact branes in representations $R, R^{\prime}$ on the external legs as in fig. 10.

Note that there is a subtle issue in this picture related to the position of the noncompact branes in the normal directions to the exceptional curve. Each conifold singularity of the hypersurface (4.8) can be written in local coordinates as

$$
u v=x y .
$$

where the local equations of the curves $\Sigma, \Sigma^{\prime}$ are $u=v=x=0$ and respectively $u=v=$ $y=0$. The small resolution $X_{\alpha}$ can be locally described by the equations

$$
u \rho=\eta y, \quad v \eta=\rho x,
$$

where $[\rho: \eta]$ are homogeneous coordinates on the exceptional curve.

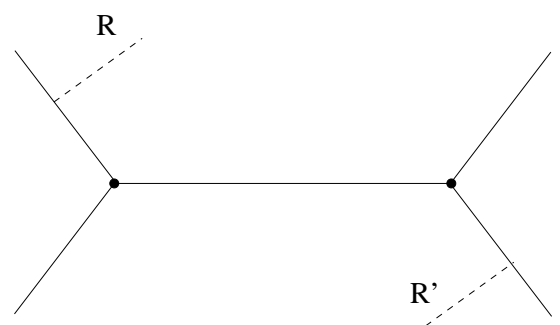

Figure 10: Local conifold geometry with noncompact branes on opposite external legs.

As expected, we recognize the above equations as the transition functions of the normal bundle $\mathcal{O}(-1) \oplus \mathcal{O}(-1)$ to the exceptional curve in $X_{\alpha}$. The strict transforms of the curves 
$\Sigma, \Sigma^{\prime}$ are locally given by the equations

$$
\Sigma: \quad u=\rho=0 \quad \Sigma^{\prime}: \quad v=\eta=0
$$

The first equation describes the fiber of the first $\mathcal{O}(-1)$ direct summand of the normal bundle over the point $\rho=0$ in $\mathbb{P}^{1}$, while the second equation describes the fiber of the second direct summand over the point $\eta=0$. Therefore the noncompact branes must be placed along different normal directions to the curve at the two fixed points of the torus action, as represented in fig. 10. The contribution of such a configuration to the partition function $[2]$ is

$$
\sum_{Q} W_{R Q} W_{Q^{t} R^{\prime}}(-1)^{l(Q)} q_{f}^{l(Q)}
$$

Gluing all individual contributions, we obtain the following final formula for the partition function of the deformed threefold

$$
Z_{d e f}=\sum_{R, R^{\prime}} q_{b}^{l(R)+l\left(R^{\prime}\right)}\left(\frac{\sum_{Q} W_{R Q} W_{Q^{t} R^{\prime}}(-1)^{l(Q)} q_{f}^{l(Q)}}{W_{R \bullet}^{2} W_{\bullet R^{\prime}}^{2}}\right)^{2 g-2}
$$

This expression is identical to the partition function (2.15) computed using the vertex formula (2.12).

For completeness we record below some low degree terms in the expansion

$$
\begin{aligned}
F= & -2\left(2 \sin \frac{g_{s}}{2}\right)^{2} q_{b}+\frac{2}{\left(2 \sin \frac{g_{s}}{2}\right)^{2}} q_{f} \\
& +4\left(2 \sin \frac{g_{s}}{2}\right)^{2} q_{b} q_{f}+\frac{2}{2\left(2 \sin g_{s}\right)^{2}} q_{f}^{2}+15\left(2 \sin \frac{g_{s}}{2}\right)^{4} q_{b}^{2}-12\left(2 \sin \frac{g_{s}}{2}\right)^{6} q_{b}^{2}+2\left(2 \sin \frac{g_{s}}{2}\right)^{8} q_{b}^{2} \\
& -2\left(2 \sin \frac{g_{s}}{2}\right)^{2} q_{b} q_{f}^{2}-60\left(2 \sin \frac{g_{s}}{2}\right)^{4} q_{b}^{2} q_{f}+34\left(2 \sin \frac{g_{s}}{2}\right)^{6} q_{b}^{2} q_{f}-4\left(2 \sin \frac{g_{s}}{2}\right)^{8} q_{b}^{2} q_{f} \\
& +90\left(2 \sin \frac{g_{s}}{2}\right)^{4} q_{b}^{2} q_{f}^{2}-44\left(2 \sin \frac{g_{s}}{2}\right)^{6} q_{b}^{2} q_{f}^{2}+5\left(2 \sin \frac{g_{s}}{2}\right)^{8} q_{b}^{2} q_{f}^{2}+\cdots
\end{aligned}
$$

Some of these predictions can be again checked using enumerative techniques similar to those in appendix $\mathrm{A}$.

\section{Further Directions and Generalizations}

There are several directions one could pursue starting from the results of the present paper. Probably the most important question at the present stage is whether this vertex formalism can be given a rigorous mathematical construction. Most likely such a construction would have to be formulated in terms of relative stable maps which already played central role in the mathematical theory of the topological vertex [18-20] and also in [6].

Another important question is whether a similar construction can be carried out for

more general Calabi-Yau threefolds. In principle it should be possible to develop a similar formalism for any noncompact Calabi-Yau manifold which admits a degenerate torus action. 
For example, such threefolds can be obtained by resolving higher genus curves of $A D E$ singularities in Calabi-Yau threefolds. The exceptional locus of the smooth crepant resolution consists of a collection of ruled surfaces intersecting along common sections according to the Dynkin diagram of the singularity. As an illustration, the resolution of an $A_{2}$ singularity is represented in fig. 11. The case considered in this paper corresponds to a curve $\Sigma$ of $A_{1}$ singularities.

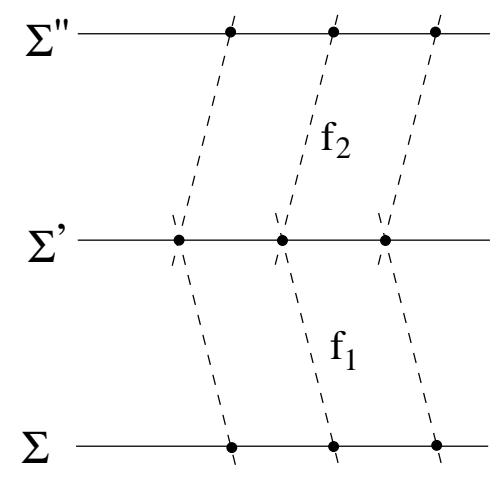

Figure 11: Exceptional locus of an $A_{2}$ singularity over a genus $g$ curve $\Sigma$.

Applying the methods developed so far, we can propose an expression for the partition function of these local threefolds. To keep this section short we will sketch some details for a curve of $A_{2}$ singularities. In this case, the basic element of our construction is a trivalent vertex which carries three representations on each leg corresponding to the three canonical sections represented in fig. 11. The vertex will have an expansion of the form

$$
V_{R_{1} R_{2} R_{3}}=\sum_{d_{1}=0}^{\infty} \sum_{d_{2}=0}^{\infty} V_{R_{1} R_{2} R_{3}}^{\left(d_{1}, d_{2}\right)} q_{f_{1}}^{d_{1}} q_{f_{2}}^{d_{2}}
$$

in terms of the exponentiated Kähler parameters of the two rulings. Moreover, by analogy with equations (3.16), (3.17), it will satisfy an identity of the form

$$
\begin{aligned}
\sum_{d_{1}=0}^{\infty} \sum_{d_{2}=0}^{\infty} \sum_{Q_{1}, Q_{2}} V_{R_{1} R_{2} R_{3}}^{\left(d_{1}, d_{2}\right)} W_{R_{1} Q_{1}} W_{Q_{1} R_{2}} W_{R_{2} Q_{2}} W_{Q_{2} R_{3}} q_{f_{1}}^{d_{1}+l\left(Q_{1}\right)} q_{f_{2}}^{d_{2}+l\left(Q_{2}\right)} \\
=\left(q^{-\kappa\left(R_{1}\right) / 2}(-1)^{l\left(R_{1}\right)}\right)^{e_{1}}\left(q^{\kappa\left(R_{2}\right) / 2}(-1)^{l\left(R_{2}\right)}\right)^{e_{1}}\left(q^{-\kappa\left(R_{3}\right) / 2}(-1)^{l\left(R_{3}\right)}\right)^{e_{2}}
\end{aligned}
$$

where $e_{1}, e_{2}$ are the degrees of the ruled surfaces satisfying $e_{1}+e_{2}=2 g-2$. In general, the trivalent vertex will carry $r+1$ representations on each leg, where $r$ is the rank of the corresponding $A D E$ group, and it will satisfy similar recursion relations. It would be very interesting to test this conjecture in this broader class of examples.

One can further construct variations by allowing jumps and global monodromy for the singularity fibration. In particular we can have chains of ruled surfaces as above with arbitrary numbers of reducible fibers. It would be very interesting to develop a coherent approach to the topological string partition function on all threefolds with degenerate torus action following the underlying principles of the present paper. This would be an important new class of exactly solvable threefolds in Gromov-Witten theory which could serve as testing ground for mirror symmetry and other ideas $[3,4,8,22,26]$ in topological string theory. 


\section{A. Enumerative Computations}

In this section we check the predictions (4.5) for nontoric curve classes by direct enumerative computations. For convenience, let us reproduce the formula (4.5) below

$$
\begin{aligned}
& q_{b} q_{1}^{-1} q_{2}^{-1} q_{3}^{-1}-q_{b}^{2} q_{1}^{-2} q_{2}^{-2} q_{3}^{-2}+q_{b}^{2}\left(q_{1}^{-1} q_{2}^{-2} q_{3}^{-2}+q_{1}^{-2} q_{2}^{-1} q_{3}^{-2}+q_{1}^{-2} q_{2}^{-2} q_{3}^{-1}\right)-q_{b}^{2}\left(q_{1}^{-2} q_{2}^{-1} q_{3}^{-1}\right. \\
& \left.+q_{1}^{-1} q_{2}^{-2} q_{3}^{-1}+q_{1}^{-1} q_{2}^{-1} q_{3}^{-2}\right)+2 q_{b} q_{f} q_{1}^{-1} q_{2}^{-1} q_{3}^{-1}+2 q_{b}^{2} q_{f}\left(q_{1}^{-2} q_{2}^{-2} q_{3}^{-1}+q_{1}^{-2} q_{2}^{-1} q_{3}^{-2}+q_{1}^{-1} q_{2}^{-2} q_{3}^{-2}\right) \\
& -2 q_{b}^{2} q_{f} q_{1}^{-2} q_{2}^{-2} q_{3}^{-2} .
\end{aligned}
$$

Let $S$ denote the three point blow-up of $\mathbb{F}_{0}$ considered in section 3.1. We will denote by $e_{1}, e_{2}, e_{3}$ the exceptional $(-1,-1)$ curves on $S$ and by $e_{1}^{\prime}, e_{2}^{\prime}, e_{3}^{\prime}$ the remaining components of the reducible fibers.

The residual Gromov-Witten free energy has the form

$$
F_{G W}=\sum_{h \geq 0} g_{s}^{2 h-2} \sum_{\beta \neq 0} C_{\beta}^{h} q^{\beta}
$$

where $\beta=n_{b} s+n_{f} f-\sum_{i=1}^{3} n_{i} e_{i}$ is a curve class on $S$.

The $C_{\beta}^{h}$ are defined by equivariant integration on the moduli space of stable maps to $S$

$$
C_{\beta}^{h}=\int_{\left[\bar{M}_{h, 0}(S, \beta)\right]^{v i r}} e_{T}(\mathcal{V}) .
$$

where $\mathcal{V}$ is the obstruction complex on the moduli space constructed as follows. Consider the diagram

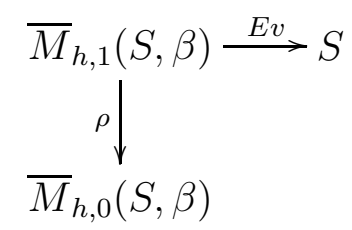

where $\bar{M}_{h, 1}(S, \beta)$ denotes the moduli space of stable maps to $S$ with one marked point, $E v: \bar{M}_{h, 1}(S, \beta) \rightarrow S$ is the evaluation map, and $\rho: \bar{M}_{h, 1}(S, \beta) \rightarrow \bar{M}_{h, 0}(S, \beta)$ is the forgetful map. Then

$$
\mathcal{V}=-R^{\bullet} \rho_{*}\left(E v^{*} K_{S}\right)
$$

where $K_{S}$ is the canonical line bundle of $S$. Note that in formula (A.2) we integrate on the moduli space of stable maps with connected domain as opposed to equation (2.2) where disconnected domains are allowed.

In some cases we can evaluate the integral (A.4) by localization with respect to the degenerate torus action in order to test the predictions (4.5). The structure of a generic stable map to $S$ fixed under the torus action is the following. The domain consists of two components ${ }^{3}$ of arbitrary genera $h_{1}, h_{2}$ and a certain number of rational components $F_{1}, \ldots, F_{k}, E_{1}, \ldots, E_{m}, E_{1}^{\prime}, \ldots, E_{m^{\prime}}^{\prime}$ which intersect $C, C^{\prime}$ as shown in the picture below.

\footnotetext{
${ }^{3}$ Note that we are using the term components somewhat loosely. In general, $C, C^{\prime}, F_{1}, \ldots, E_{m^{\prime}}^{\prime}$ will have several irreducible components.
} 


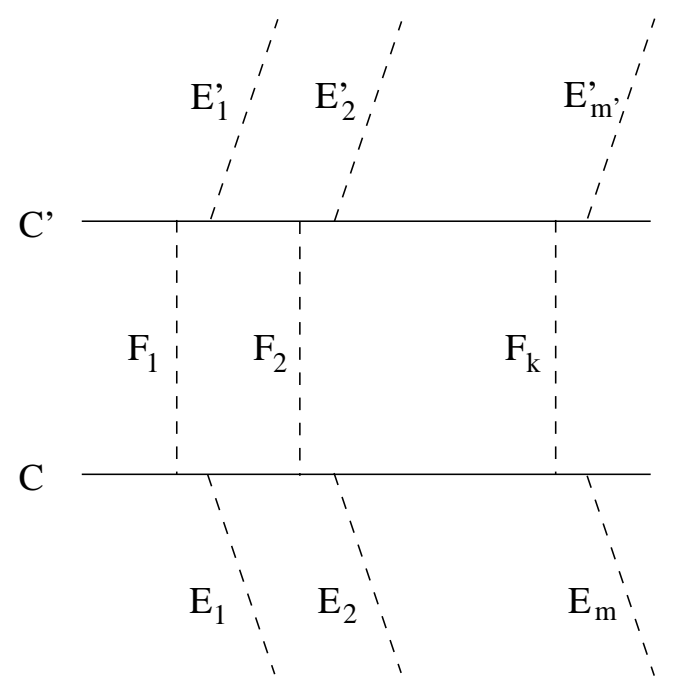

Figure 12: Domain of a generic map.

The higher genus components $C, C^{\prime}$ are mapped to the canonical sections $\Sigma, \Sigma^{\prime}$, and the rational components $F_{1}, \ldots, F_{k}$ are mapped to fibers of $S$. The rational components $E_{1}, \ldots, E_{m}, E_{1}^{\prime}, \ldots, E_{m}^{\prime}$ are mapped to $e_{1}, e_{2}, e_{3}$ and respectively $e_{1}^{\prime}, e_{2}^{\prime}, e_{3}^{\prime}$.

The contribution of a generic fixed locus to the integral (A.6) is quite complicated and cannot be evaluated by localization. However, localization can be applied in some special cases, and we will discuss two such situations for illustrations. Let us consider the terms

$$
q_{b}^{2} q_{1}^{-1} q_{2}^{-2} q_{3}^{-2}-2 q_{b}^{2} q_{f} q_{1}^{-2} q_{2}^{-2} q_{3}^{-2}
$$

in (4.5), which correspond to classes of the form $2[\Sigma]+e_{1}$ and respectively $2[\Sigma]+f$.

These two cases exhibit a very similar fixed locus structure. There is one fixed locus $\Xi$ isomorphic to the moduli space $\bar{M}_{0,1}\left(\mathbb{P}^{1}, 2\right)$ of degree two genus zero stable maps to $\mathbb{P}^{1}$ with one marked point. Let us concentrate on the second case $\beta=2[\Sigma]+f$. The domain of a fixed map to $S$ consists of two rational components $C, F$ which are are mapped to $\Sigma$ and to a fiber of $S$ with degrees 2 and 1 respectively.

The contribution of such a fixed locus to the integral (A.2) takes the form

$$
\int_{\left[\bar{M}_{0,1}(\Sigma, 2)\right]^{v i r}} \frac{e_{T}(\mathcal{V})}{e_{T}\left(N_{\Xi}^{v i r}\right)}
$$

where $N_{\Xi}^{\text {vir }}$ is the virtual normal bundle to $\Xi$. The integrand in this expression can be evaluated using standard normalization sequence techniques. Then we obtain an expression of the form

$$
\frac{e_{T}(\mathcal{V})}{e_{T}\left(N_{\Xi}^{v i r}\right)}=e_{T}\left(\mathcal{V}_{0}\right) e_{T}\left(\mathcal{V}_{F}\right) e_{T}(\text { node })
$$

where $e_{T}\left(\mathcal{V}_{0}\right) \in H^{*}\left(\bar{M}_{0,0}\left(\mathbb{P}^{1}, 2\right)\right)$ is the obstruction class in the local theory of the curve $\Sigma[6]$ pulled back to $\bar{M}_{0,1}\left(\mathbb{P}^{1}, 2\right)$. $e_{T}\left(\mathcal{V}_{F}\right)$ encodes the contribution of the rational components $F$, and $e_{T}$ (node) represents the contribution of the node. 
For completeness recall [6] that that $e_{T}\left(\mathcal{V}_{0}\right)$ is defined as

$$
e_{T}\left(\mathcal{V}_{0}\right)=e_{T}\left(-R^{\bullet} \rho_{*}\left(e v^{*} L_{1}\right)\right) e_{T}\left(-R^{\bullet} \rho_{*}\left(e v^{*} L_{2}\right)\right)
$$

where $L_{1} \oplus L_{2}$ is the normal bundle to the target curve $\Sigma$ in the threefold, and the maps ev $: \bar{M}_{h, 1}\left(\Sigma, n_{b}\right) \rightarrow \Sigma, \rho: \bar{M}_{h, 1}\left(\Sigma, n_{b}\right) \rightarrow \bar{M}_{h, 0}\left(\Sigma, n_{b}\right)$ are standard. In our case, the normal bundle to $\Sigma$ in $X$ is

$$
N_{X}(\Sigma)=\mathcal{O}(-3) \oplus \mathcal{O}(1)
$$

and $T$ acts with opposite weights on the two direct summands. Therefore we have

$$
e_{T}\left(\mathcal{V}_{0}\right)=\frac{e_{T}\left(R^{1} \rho_{*} e v^{*}(\mathcal{O}(-3))\right)}{e_{T}\left(R^{0} \rho_{*} e v^{*}(\mathcal{O}(1))\right)}
$$

The contribution of the node is also standard

$$
e_{T}(\text { node })=\frac{-\lambda^{2}}{\lambda(\lambda-\psi)}
$$

where $-\lambda$ is weight of the torus action along the fiber of $S$ and $\psi \in H^{*}\left(\bar{M}_{0,1}\left(\mathbb{P}^{1}, 1\right)\right)$ is the Mumford class.

In order to evaluate $e_{T}\left(\mathcal{V}_{F}\right)$ let us determine the bundle $\mathcal{V}_{F}$ over $\bar{M}_{0,1}\left(\mathbb{P}^{1}, 2\right)$. The fiber of $\mathcal{V}_{F}$ over a point $\left(C, x_{1}, f_{0}\right)$ is the vector space

$$
H^{1}\left(F, f_{1}^{*} K_{S}\right)
$$

where $f_{0}: C \rightarrow S, f_{1}: F_{1} \rightarrow S$ denote the restriction of the stable map to $C$ and respectively $F_{1}$. Note that $f_{0}$ factorizes through the section $\Sigma \rightarrow S$ and $f_{1}$ factorizes through the fiber $S_{f_{0}\left(x_{1}\right)}$ of $S$.

From the definition, it follows that

$$
\mathcal{V}_{F}=e v^{*}\left(R^{1} \pi_{*}\left(K_{S}\right)\right)
$$

where $e v: \bar{M}_{0,1}(\Sigma, 2) \rightarrow \Sigma$ is the evaluation map, and $\pi: S \rightarrow \Sigma$ is the natural projection. Using the base change theorem, we can compute

$$
R^{1} \pi_{*}\left(K_{S}\right)=K_{\Sigma}
$$

therefore we find

$$
\mathcal{V}_{F}=e v^{*}\left(K_{\Sigma}\right)
$$

Collecting the intermediate results (A.8) and (A.11), it follows that the integral (A.6) becomes

$$
\int_{\left[\bar{M}_{0,1}\left(\mathbb{P}^{1}, 2\right)\right]_{T}^{v i r}} e_{T}\left(e v^{*}\left(K_{\Sigma}\right)\right) \frac{e_{T}\left(R^{1} \rho_{*} e v^{*}(\mathcal{O}(-3))\right)}{e_{T}\left(R^{0} \rho_{*} e v^{*}(\mathcal{O}(1))\right)} \frac{-\lambda^{2}}{\lambda(\lambda-\psi)}
$$

Let us denote by $c_{i}, i=0,1, \ldots$ the nonequivariant Chern classes of $R^{1} \rho_{*} e v^{*}(\mathcal{O}(-3))$ and by $c_{i}^{\prime}, i=0,1, \ldots$ the nonequivariant Chern classes of $R^{0} \rho_{*} e v^{*}(\mathcal{O}(1))$. Then a short computation shows that (A.12) reduces the nonequivariant integral

$$
\int_{\left[\bar{M}_{0,1}\left(\mathbb{P}^{1}, 2\right)\right]^{v i r}} e\left(e v^{*}\left(K_{\Sigma}\right)\right)\left[c_{1} c_{1}^{\prime}+\left(c_{1}^{\prime}\right)^{2}+c_{2}-c_{2}^{\prime}+\left(c_{1}+c_{1}^{\prime}\right) \psi+\psi^{2}\right]
$$


This integral can be evaluated by localization with respect to a torus action induced by the standard torus action on $\mathbb{P}^{1}$. Applying the divisor axiom [7, Chapter 10], we have

$$
\begin{aligned}
& \int_{\left.\left[\bar{M}_{0,1}\left(\mathbb{P}^{1}, 2\right)\right]\right]^{v i r}} e\left(e v^{*}\left(K_{\Sigma}\right)\right)\left[c_{1} c_{1}^{\prime}+\left(c_{1}^{\prime}\right)^{2}+c_{2}-c_{2}^{\prime}\right] \\
& =\int_{2[\Sigma]} e\left(K_{\Sigma}\right) \int_{\left.\left[\bar{M}_{0,0}\left(\mathbb{P}^{1}, 2\right)\right]\right]^{v i r}}\left[c_{1} c_{1}^{\prime}+\left(c_{1}^{\prime}\right)^{2}+c_{2}-c_{2}^{\prime}\right] \\
& =(-4) \int_{\left[\bar{M}_{0,0}\left(\mathbb{P}^{1}, 2\right)\right]^{v i r}}\left[c_{1} c_{1}^{\prime}+\left(c_{1}^{\prime}\right)^{2}+c_{2}-c_{2}^{\prime}\right] .
\end{aligned}
$$

The integral over $\bar{M}_{0,0}\left(\mathbb{P}^{1}, 2\right)$ can be evaluated using localization as explained in [7, Chapter 9]. We obtain

$$
\int_{\left[M_{0,0}\left(\mathbb{P}^{1}, 2\right)\right]^{v i r}}\left[c_{1} c_{1}^{\prime}+\left(c_{1}^{\prime}\right)^{2}+c_{2}-c_{2}^{\prime}\right]=\frac{7}{8} .
$$

Next, the integral

$$
\int_{\left.\left[\bar{M}_{0,1}\left(\mathbb{P}^{1}, 2\right)\right]\right]^{i r}} e\left(e v^{*}\left(K_{\Sigma}\right)\right)\left[\left(c_{1}+c_{1}^{\prime}\right) \psi+\psi^{2}\right]
$$

can also be evaluated by localization on $\bar{M}_{0,1}\left(\mathbb{P}^{1}, 2\right)$. The result is

$$
\int_{\left[\bar{M}_{0,1}\left(\mathbb{P}^{1}, 2\right)\right]^{v i r}} e\left(e v^{*}\left(K_{\Sigma}\right)\right)\left[\left(c_{1}+c_{1}^{\prime}\right) \psi+\psi^{2}\right]=\frac{3}{2} .
$$

Collecting the intermediate results, the result of the integral (A.13) is

$$
(-4) \times \frac{7}{8}+\frac{3}{2}=-2
$$

in agreement with the prediction (A.5).

The first invariant in (A.5) can be computed in a similar manner. The domain of a fixed map consists again of two components $C, E$ which are mapped to $\Sigma$ and $e_{1}$ with degrees 2 and 1 respectively. Therefore the fixed locus is isomorphic to $\bar{M}_{0,1}\left(\mathbb{P}^{1}, 2\right)$, except that the obstruction class is different. Since $E$ is mapped to the rigid $(-1,-1)$ curve $e_{1}$, the marked point $x_{1} \in C$ must be mapped to the intersection point $p_{1}$ of $\Sigma$ and $e_{1}$. This constraint can be enforced by replacing the factor $e_{T}\left(e v^{*}\left(K_{\Sigma}\right)\right)$ in (A.13) by $e v^{*}\left(\omega_{p_{1}}\right)$, where $\omega_{p_{1}}$ is the equivariant class of the point $p_{1} \in C$. Furthermore, we can write

$$
\omega_{p_{1}}=e_{T}\left(\mathcal{O}_{\Sigma}(1)\right)
$$

therefore the contribution of the fixed locus becomes

$$
\int_{\left.\left[\bar{M}_{0,1}\left(\mathbb{P}^{1}, 2\right)\right]\right]^{v i r}} e\left(e v^{*}\left(\mathcal{O}_{\Sigma}(1)\right)\right)\left[c_{1} c_{1}^{\prime}+\left(c_{1}^{\prime}\right)^{2}+c_{2}-c_{2}^{\prime}+\left(c_{1}+c_{1}^{\prime}\right) \psi+\psi^{2}\right] .
$$

This integral can be evaluated as above by localization. Since $\mathcal{O}_{\Sigma}(1) \simeq K_{\Sigma}^{-1 / 2}$, the computation is in fact identical to the previous one, except for an overall $-\frac{1}{2}$ factor. Therefore in this case the final result is

$$
-\frac{1}{2} \times(-2)=1 \text {, }
$$

in agreement with (A.13). 


\section{References}

[1] M. Aganagic, M. Mariño and C. Vafa, "All Loop Topological String Amplitudes from Chern-Simons Theory", Commun. Math. Phys. 247 (2004) 467, hep-th/0206164.

[2] M. Aganagic, A. Klemm, M. Mariño and C. Vafa, "The Topological Vertex", Commun. Math. Phys. 254 (2005) 425, hep-th/0305132.

[3] M. Aganagic, R. Dijkgraaf, A. Klemm, M. Mariño and C. Vafa, "Topological Strings and Integrable Hierarchies", hep-th/0312085.

[4] M. Aganagic, H. Ooguri, N. Saulina and C. Vafa, "Black Holes, $q$-Deformed $2 D$ Yang-Mills and Nonperturbative Topological Strings", hep-th/0411280.

[5] M. Aganagic, A. Neitzke and C. Vafa, "BPS Microstates and the Open Topological String Wave Function", hep-th/0504054.

[6] J. Bryan and R. Pandharipande, "The Local Gromov-Witten Theory of Curves", math.AG/0411037.

[7] D. Cox and S. Katz, Mirror Symmetry and Algebraic Geometry, Mathematical Surveys and Monographs Vol. 68, AMS, Providence, RI (1999).

[8] A. Dabholkar, F. Denef, G. W. Moore and B. Pioline, "Exact and Asymptotic Degeneracies of Small Black Holes", hep-th/0502157.

[9] D.-E. Diaconescu, B. Florea and A. Grassi, "Geometric Transitions and Open String Instantons", Adv. Theor. Math. Phys. 6 (2002) 619, hep-th/0205234.

[10] D.-E. Diaconescu, B. Florea and A. Grassi, "Geometric Transitions, del Pezzo Surfaces and Open String Instantons", Adv. Theor. Math. Phys. 6 (2002) 643, hep-th/0206163.

[11] D.-E. Diaconescu and B. Florea, "Large $N$ Duality for Compact Calabi-Yau Threefolds", hep-th/0302076.

[12] D.-E. Diaconescu and B. Florea, "Localization and Gluing of Topological Amplitudes", Commun. Math. Phys. 257 (2005) 119, hep-th/0309143.

[13] D.-E. Diaconescu, R. Dijkgraaf, R. Donagi, C. Hofman and T. Pantev, "Geometric Transitions and Integrable Systems", hep-th/0506196.

[14] R. Hartshorne, Algebraic Geometry, Springer-Verlag (1993).

[15] A. Iqbal and A.-K. Kashani-Poor, "Instanton Counting and Chern-Simons Theory", Adv. Theor. Math. Phys. 7 (2003) 459, hep-th/0212279.

[16] A. Iqbal and A.-K. Kashani-Poor, " $S U(N)$ Geometries and Topological String Amplitudes", hep-th/0306032. 
[17] S. Katz, D. R. Morrison and R. Plesser, "Enhanced Gauge Symmetry in Type II String Theory", Nucl. Phys. B477 (1996) 74, hep-th/9601108.

[18] C.-C. M. Liu, K. Liu and J. Zhou, "A Proof of a Conjecture of Mariño-Vafa on Hodge Integrals", J. Diff. Geom. 65 (2004) 289, math. AG/0306434.

[19] C.-C. M. Liu, K. Liu and J. Zhou, "A Formula of Two-Partition Hodge Integrals", math.AG/0310272.

[20] J. Li, C.-C. M. Liu, K. Liu and J. Zhou, "A Mathematical Theory of the Topological Vertex", math.AG/0408426.

[21] A. Okounkov and R. Pandharipande, "Hodge Integrals and Invariants of the Unknot", Geom. Topol. 8 (2004) 675, math. AG/0307209.

[22] H. Ooguri, A. Strominger and C. Vafa, "Black Hole Attractors and the Topological String", hep-th/0405146.

[23] B. Szendröi, "Calabi-Yau Threefolds with a Curve of Singularities and Counterexamples to the Torelli Problem," math.AG/9901078.

[24] B. Szendrői, "Artin Group Actions on Derived Categories of Threefolds," math. AG/0210121.

[25] B. Szendrői, "Enhanced Gauge Symmetry and Braid Group Actions", Commun. Math. Phys. 238 (2003) 35, math. AG/0210122.

[26] C. Vafa, "Two Dimensional Yang-Mills, Black Holes and Topological Strings", hep-th/0406058.

[27] E. Witten, "Chern-Simons Gauge Theory as a String Theory", The Floer Memorial Volume, H. Hofer, C.H. Taubes, A. Weinstein and E. Zehnder, eds, Birkhäuser (1995) 637, hep-th/9207094. 